\title{
Universat-SOCRAT multi-satellite project to study TLEs and TGFs
}

\author{
Mikhail Panasyuk 1,2, Pavel Klimov ${ }^{1 *}$ (1), Sergei Svertilov ${ }^{1,2}$, Alexander Belov ${ }^{1,2}$, Vitali Bogomolov1,2, \\ Andrei Bogomolov' , Gali Garipov' , Anatoly lyudin' ', Margarita Kaznacheeva', Ivan Maksimov', \\ Alexander Minaev ${ }^{1,2}$, Artem Novikov ${ }^{1,2}$, Pavel Minaev ${ }^{3}$, Vasili Petrov ${ }^{1}$, Alexei Pozanenko ${ }^{3,4}$, Yan Shtunder ${ }^{1,2}$ \\ and Ivan Yashin ${ }^{1}$
}

\begin{abstract}
We present concept of a new multi-satellite Universat-SOCRAT project aimed to study transient phenomena in the upper atmosphere such as transient luminous events (TLEs) and terrestrial gamma-ray flashes (TGFs). It is a new space project of Lomonosov Moscow State University based on the use of a few satellites in the near-Earth orbit for real-time monitoring of radiation environment, natural (asteroids, meteoroids) and artificial (space debris) potentially dangerous objects, electromagnetic transients including cosmic gamma-ray bursts, terrestrial gamma-ray flashes, and optical and ultraviolet bursts in the Earth's atmosphere.

Study of TLEs and TGFs remains an important and demanding task despite of a multitude of recently acquired data for these phenomena. This might be explained by the absence of comprehensive theoretical understanding of physical nature of high-energy processes in the Earth's atmosphere. Multi-wavelength synchronous observations with moderate accuracy of localization of TGF and TLE events are necessary to gain an insight of physics governing these high-energy processes in the Earth's atmosphere. In the article, we present results of TLE observations in space experiments of Moscow State University and discuss advanced instruments for optical observations of TLEs, as well as gamma-ray burst monitor and tracking gamma spectrometer for TGFs observations.
\end{abstract}

Keywords: Electromagnetic transients, TLE, TGF, Imager, Track spectrometer

\section{Introduction}

One of the main problems of modern geoscience and atmospheric physics in particular is understanding the nature of the so-called transient atmospheric events (TAE), which include terrestrial gamma-ray flashes (TGFs) and transient luminous events (TLEs). TGFs and TLEs are observed both in the stratosphere and mesosphere, i.e., at the source altitude from about $12 \mathrm{~km}$ up to a few dozen kilometers (Cummer et al. 2014). Electromagnetic transient phenomena in the upper atmosphere are global in nature and associated with electric discharges. During the electric discharges between the clouds and the clouds and ionosphere (altitudes from $5-10 \mathrm{~km}$ to 60 $90 \mathrm{~km}$ ) short-time bursts of electromagnetic radiation,

\footnotetext{
*Correspondence: pavel.klimov@gmail.com

'Skobeltsyn Institute of Nuclear Physics, Lomonosov Moscow State University, 1(2), Leninskie gory, Moscow 119991, Russia

Full list of author information is available at the end of the article
}

with duration from fractions of millisecond up to several hundred of milliseconds, within wide spectral range from visual light up to UV, and even X-rays and gamma-rays, are observed.

TLEs are optical flashes known as sprites, elves, and blue jets. Their parameters including spatial and temporal structure, rate of occurrence, and optical brightness in different ranges could be found in (Vaughan and Vonnegut 1989; Fisher 1990; Lyons 1994; Boeck et al. 1995; Winckler et al. 1996; Boccippio et al. 1995).

TGFs are extremely short gamma-ray events, which were discovered by BATSE experiment on-board Compton Gamma-Ray Observatory ((Fishman et al. 1994)). Since 1991 up to 2000, 36 TGFs were discovered, some of them were associated with thunderstorms. Later, these observations were confirmed by the space observatory RHESSI (Smith et al. 2005). The largest TGF statistics was obtained by Fermi space gamma observatory that was launched in 2008. To the present, more than 2700 
TGFs have been detected in the $10 \mathrm{keV}-40 \mathrm{MeV}$ energy range, the average detecting frequency is one burst in $\sim 4$ days (Briggs et al. (2013); Roberts et al. (2018)). The TGFs were also studied during AGILE (Tavani et al. (2011)), and INTEGRAL (Minaev et al. 2014; Minaev and Pozanenko 2018 and Vernov (Bogomolov et al. 2017)) space missions.

TGFs and TLEs might be the consequence of physical processes resulting from different kinds of highenergy release over short time intervals (between $10^{-6}$ and $10^{-3} \mathrm{~s}$ ). It is supposed that TGFs could accompany a particular type of a lightning activity (upward positive intra-cloud (IC) lightning) (Dwyer et al. (2012)). The other manifestation of energetic processes during thunderstorm activity is upward travelling of relativistic run-away electron beams at the altitudes of $60-80 \mathrm{~km}$ (Bell et al. (1995); Lehtinen et al. (1997)). Electrons with energies of $\sim 1 \mathrm{MeV}$ and higher are able to penetrate through the upper atmosphere, up to the Earth's magnetosphere, and finally into radiation belts. However, attempts of direct measurements of runaway electrons by means of large area electron detector on-board Tatiana-2 satellite did not show any tangible results (Sadovnichy et al. (2011)). Nevertheless, detection of electrons from the CGRO/BATSE (Dwyer et al. (2008)) and positrons from the GBM/Fermi (Briggs et al. 2011) space missions may be considered as indirect evidence of the occurrence of runaway electron avalanches taking place in the area of thunderstorm activity. According to these results, positron portion can reach up to $11 \%$ of these up-going beams by different estimations (Briggs et al. (2011)).

So despite more than 20 years of experimental and theoretical research of TAE, there are no clear interpretations of such phenomena. Study of TLEs and TGFs is very important for understanding of their physical nature and verification of theoretical models. Simultaneous observations at different electromagnetic wave bands with, at least, moderately good accuracy of event localization are necessary to gain an insight of physics governing these high-energy processes in the Earth's atmosphere. Also multi-messenger observation, i.e., detection of neutrons, electrons probably connected with TGF source, can provide us direct information about acceleration mechanisms in the TGF source. As for TLEs, most of such phenomena are well-known as a specific kind of thunderstorm activity. Nevertheless, some events were observed far from thunderstorm areas. The nature of such non-usual transients is still unknown, thus further observations of TLEs are also necessary.

In this article, we present concept of a new multisatellite Universat-SOCRAT space project of Lomonosov Moscow State University based on the use of a few satellites in the near-Earth space for real-time monitoring of radiation environment, natural (asteroids, meteoroids) and artificial (space debris) potentially dangerous objects, electromagnetic transients including cosmic gamma-ray bursts, terrestrial gamma-ray flashes, and optical and ultraviolet bursts in the Earth's atmosphere.

\section{The problem of TLE and TGF study in modern space missions}

The common point is that transient atmospheric events are caused by discharges related to thunderstorm activity. Thus, most frequent TLEs-elves - are caused by the electromagnetic pulse (EMP) from lightning or powerful intracloud discharge. Another type of TLE-sprites-is a mesospheric phenomenon associated usually with positive cloud-to-ground lightning discharge, and is caused by a quasi-electrostatic field ((Pasko et al. 2012)).

The model of relativistic runaway electron avalanches (RREA) based on the runaway electron breakdown (REB), predicted in 1992 and studied theoretically in details by (Gurevich et al. 1992) and (Dwyer et al. 2012), is often used for interpretation of TGF. The typical feature of REB is its occurrence at electric fields lower than typical breakdown field in the cloud, with the seed electrons presence, that are necessary for its initiation. An alternative approach supposes electron multiplication during its acceleration, which is considered within the relativistic feedback discharge (RFD) model (Dwyer (2007)). This model assumes acceleration of thermal electrons up to relativistic energies that needs a very high accelerating electric field $\sim 280 \mathrm{kV} / \mathrm{cm}$. It is possible for run-away electrons produced by lightning current pulse in a strong field near lightning step-leader streamer and edges ((Moos and Moos 2006)). Due to a large number $\left(\sim 10^{12}\right)$ of cold electrons, relatively small $\left(\sim 10^{5}-10^{6}\right)$ multiplication factor in avalanche is sufficient for gamma-ray flash generation. Duration of coronal streamer discharge on the one step of lightning leader is very short $(\sim 10 \mu \mathrm{s})$; however, due to Compton scattering duration of gamma-ray flashes at satellite, altitudes can be $\sim 50 \mu s$ (Østgaard et al. (2008)).

Current experimental data on discharges in the upper atmosphere have shown that these phenomena are global. A number of discharges and the energy released in these discharges are so high that we can expect certain correlation between the discharges and other geophysical phenomena. Physical mechanisms leading to TGFs and TLEs at optical and gamma-ray ranges can be different. The Universitetsky-Tatiana, Tatiana-2, and Vernov mission data demonstrated cogently the appearance of TLEs types, which are not associated with thunderstorm activity (Sadovnichy et al. (2011); Sadovnichy et al. (2007); Klimov et al. (2018)). Below we present the results of TLE observations during these experiments and discuss the problem of TLE and TGF study during modern space missions. 


\section{Results of TLE observations in space experiments of Moscow State University}

UV fluorescence detectors were put on boards of several MSU micro-satellites in order to understand the transient events as a geophysical problem of global electric circuit better. These include "UniversitetskyTatiana”(Sadovnichy et al. (2007); Garipov et al. (2005)), "Universitetsky-Tatiana-2"(Sadovnichy et al. (2011); Vedenkin et al. (2011); Garipov et al. (2013)), and "Vernov"(Panasyuk et al. 2016a,b).

These satellites were equipped with a simple wide-field UV (240-400 nm) and near infrared (NIR) (600-800 nm) detectors (DUV). Detectors consist of two photomultiplier tubes (PMT) Hamamatsu R1463 with multi-alkali cathode, UV transparent entrance window, and electronics module, which allows to observe transient flash profiles. The field of view (FOV) $16^{\circ}$ of every detector is limited by a collimator. The detectors provided measurements of atmospheric emission with $1 \mathrm{~ms}$ (Tatiana-2) and $0.5 \mathrm{~ms}$ (Vernov) temporal resolution. The detailed description for DUV electronic module and measurements is presented in (Sadovnichy et al. 2007) and (Garipov et al. 2006). For observation from space, transient UV events generated in the upper atmosphere are at favorable conditions, as they are less absorbed in route from event to detector.

Data from DUV are registered as UV temporal profiles from transients observed from nadir direction within $16^{\circ} \mathrm{FOV}$. They demonstrate a variety of temporal structures ranging from a single peak to quite complicated profiles. Short events with about $1 \mathrm{~ms}$ duration are the most numerous and can be associated with elves, while series of flashes can be interpreted as multiple lightning discharges, or sequences of lightning and TLEs (sprites, jets, etc.). Number of events without NIR signal at all were observed. These dim transients, which have only signal in UV range, might be discharges at the "pre-lightning" stage, which do not always develop to the lightning stage, or, alternatively, can be low-altitude TLEs, like blue jets and blue starters (Klimov et al. (2017)).

Other events that have signal in both UV and NIR bands belong to the transients as well, but could not be associated with lightning itself. This conclusion is based on temporal structure of events and their spectrum that is different from the lightning spectrum (measured in (Orville and Henderson 1984) and are more like the sprite spectrum (Milikh et al. (1998)). The analyses of event altitude based on UV to IR ratio in these experiments were made by (Garipov et al. 2013) and (Klimov et al. 2017). For more reliable conclusions on the nature of luminous transients, the study of lightning and of other types TAE spectra is needed, especially in UV range.

As was earlier mentioned by (Garipov et al. 2013), some events detected on-board Tatiana-2 have occurred not above clouds, and rather far from the thunderstorm regions. The recent analyses of such events by instruments on-board Vernov satellite was made by Klimov et al. 2018, where these events were compared with the data of ground-based lightning location networks and with satellite data of the cloud coverage, and as a result of this analysis, a new class of far-from-thunderstorm events was suggested. But no hypotheses of their nature was put forward, or even suggested. More information on the spectral and spatial distributions of such events is needed in order to understand their origin and nature.

Important and new result is that in both experiments Tatiana-2 and Vernov, TAE from the large thunderstorm regions were regularly observed as a series of events registered in every period $T$, technically the smallest possible period between registered events. An example of such series detected by DUV on-board the Vernov satellite is shown in Fig. 1. Here, values of maximum signal in one bin of the oscilloscope trace, triggered every $4.5 \mathrm{~s}$, are shown. Blue and red lines correspond to UV and NIR wave bands, respectively. The average rate of lightning available from LIS (lightning imaging sensor) data (Christian et al. 1999) for the thunderstorm region is much lower than the observed transient rate in the series. Moreover, in some series, TAE are observed in clear atmosphere, no clouds conditions, which is speaking against lightning origin of those TAE. Analysis of correlation between clouds and any TAE (in series or not) is also contradict to the lightning origin of some TAE. An equal number of transients from both cloudy and cloudless areas of the atmosphere (Vedenkin et al. 2011; Garipov et al. 2013) were observed, while lightning is strongly correlated with clouds. Those data should be confirmed by new measurements with better identification of TAE and lightning.

Recently, a new instrument-detector TUS-was launched on-board the Lomonosov spacecraft. This detector is a mirror telescope with a large aperture $\left(2 \mathrm{~m}^{2}\right)$ which provides a high sensitivity of the detector. It is important to note that it has a spatial resolution of $5 \mathrm{~km}$ in the atmosphere observing the total area of $80 \times 80 \mathrm{~km}^{2}$. The detailed description of the TUS detector is presented in (Klimov et al. 2017) and first results of measurements in (Khrenov et al. 2017).

The TUS detector can be operated in four modes selected to detect various fast optical phenomena that happen in the atmosphere at different time scales and with different time sampling. The main mode has a time sampling of $0.8 \mu \mathrm{s}$. This mode is efficient for elve measurements, the most common type of transient luminous events. Three other modes have time sampling of $25.6 \mu \mathrm{s}$ and $0.4 \mathrm{~ms}$ for studying TLEs of different kinds slower than elves, namely, for sprites, blue jets, gigantic jets, etc., and $6.6 \mathrm{~ms}$ for detecting thunderstorm activity at a longer time scale of $\sim 1.7 \mathrm{~s}$. 


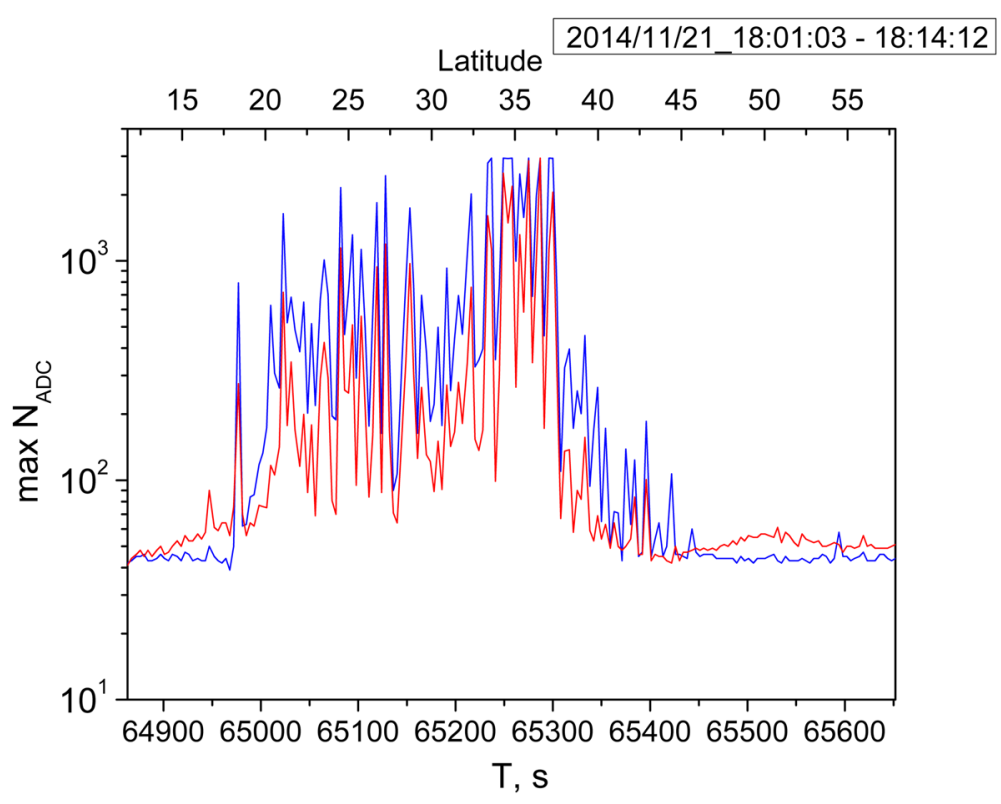

Fig. 1 Example of series of TAE in the Vernov experiment (blue curve_UV emissions; red_infrared one)

The advantage of the detector is that its spatial resolution allows us to identify some types of TLEs, for example, elves. An arc-like shape of the track made by the brightest PMTs can be seen in Fig. 2, and short duration of the signal (waveforms in Fig. 3) prove the hypotheses that this event corresponds to elve detection.

Another interesting result for the serial flashes was observed in the fourth timing mode. The example of such series is shown in Fig. 4. It has a long waveform of $1.7 \mathrm{~s}$ duration with a few dozen of peaks. This sequence of flashes has very complicated temporal structure with numerous peaks and was detected from a thunderstorm region. It is interesting to note that similar series were observed far from the thunderstorm regions as well, but peaks for these series have longer duration. Analysis of these data is still in progress.

TUS detected TLEs demonstrate the advantages of using detector with high temporal resolution, spatial resolution, also capable to record a long time series. Unfortunately, due to Lomonosov telemetry limits, the detector

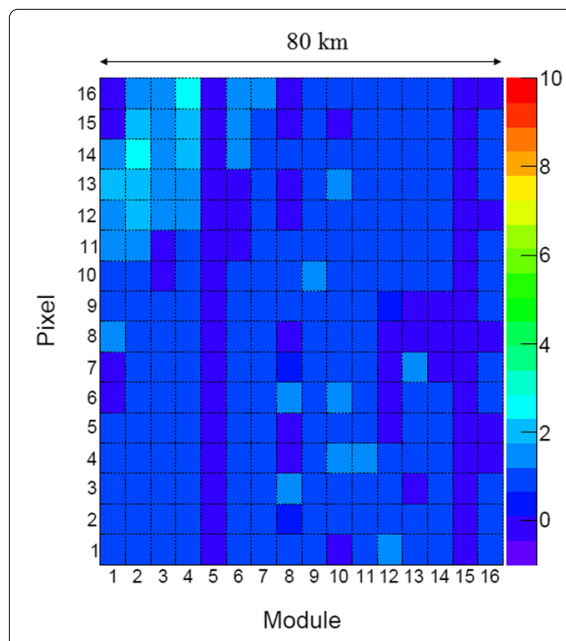

(a)

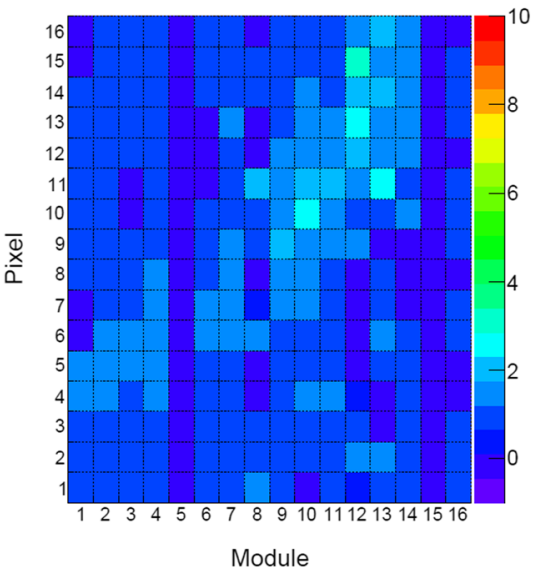

(b)

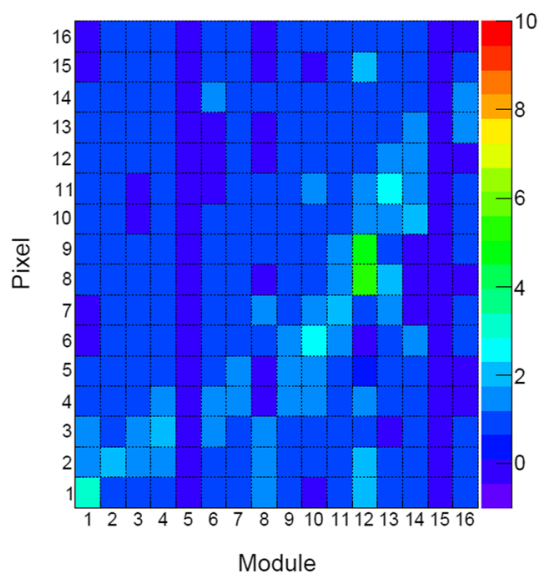

(c)

Fig. 2 Snapshots of the focal plane show arc-like shape and movement of the pattern through the detector's field of view. The snapshots were taken at $t=0.077 \mathrm{~ms}(\mathbf{a}), 0.174 \mathrm{~ms}(\mathbf{b})$, and $0.182 \mathrm{~ms}(\mathbf{c})$ from the beginning of the record. The signal amplitude is color coded in arbitrary units and scaled to individual PMT gain 


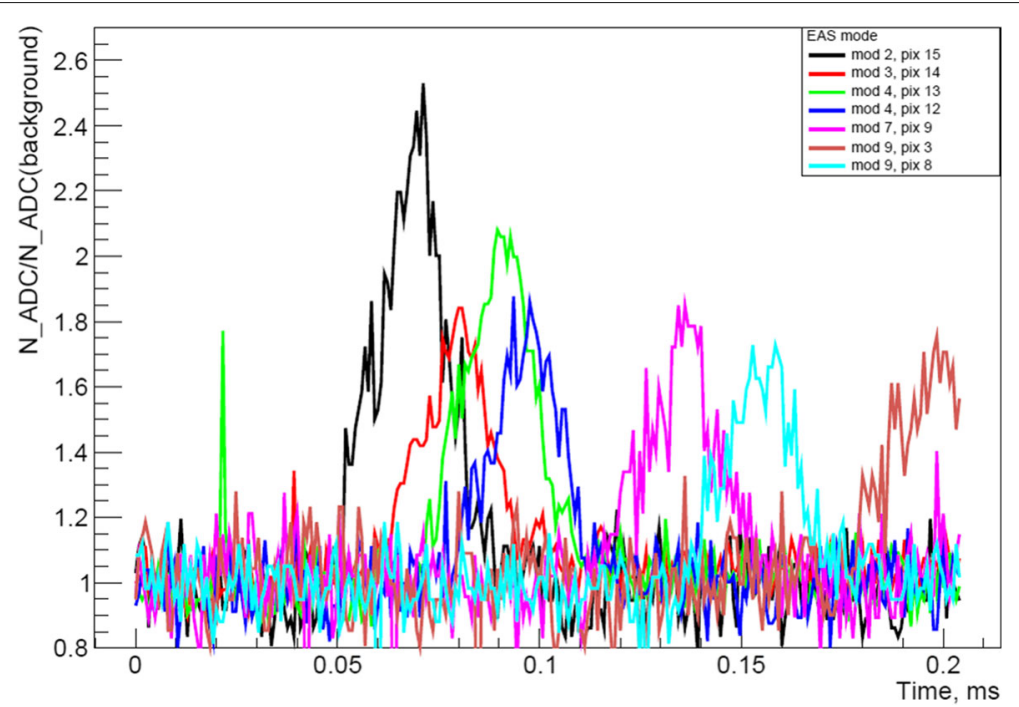

Fig. 3 Waveforms of several hit pixels of the elves type event registered on September 18, 2016 above continental Africa. The $Y$-axis is a ratio of ADC counts and background signal for each pixel

can record only one event per minute, having a huge dead time between events, and therefore, four modes with different temporal resolutions were used separately and not simultaneously.

\section{The TLE and TGF study in other missions}

At least three space projects TARANIS (Blanc et al. (2007)), ASIM (Neubert et al. (2006)), and TRYAD ((Briggs et al. 2015)) are intended for complex studies of the high-energy phenomena in the atmosphere, including TLEs and TGFs. The ASIM mission was successfully launched in April 2018, while TARANIS and TRYAD should be realized in the near future.
The TARANIS (Tool for the Analysis of RAdiations from lightNIng and Sprites) project with its claimed goals, tasks, and methods is very similar to the Russian space mission Vernov that was operated in space during the second half of 2014 (Panasyuk et al. 2016a,b). TARANIS is planned for launch in 2019 to the solar-synchronous $700 \mathrm{~km}$ high circular orbit. The main goal of this mission is to study TGFs and TLEs in a wide wavelength range from radio to gamma (Blanc et al. (2007)). The main instrument of TARANIS is XGRE designed to detect TGFs and accompanied electron beams, so-called TEBs (Sarria et al. (2017)). XGRE consists of three scintillating detectors using $\mathrm{LaBr}_{3}(\mathrm{Ce})$, with different observation

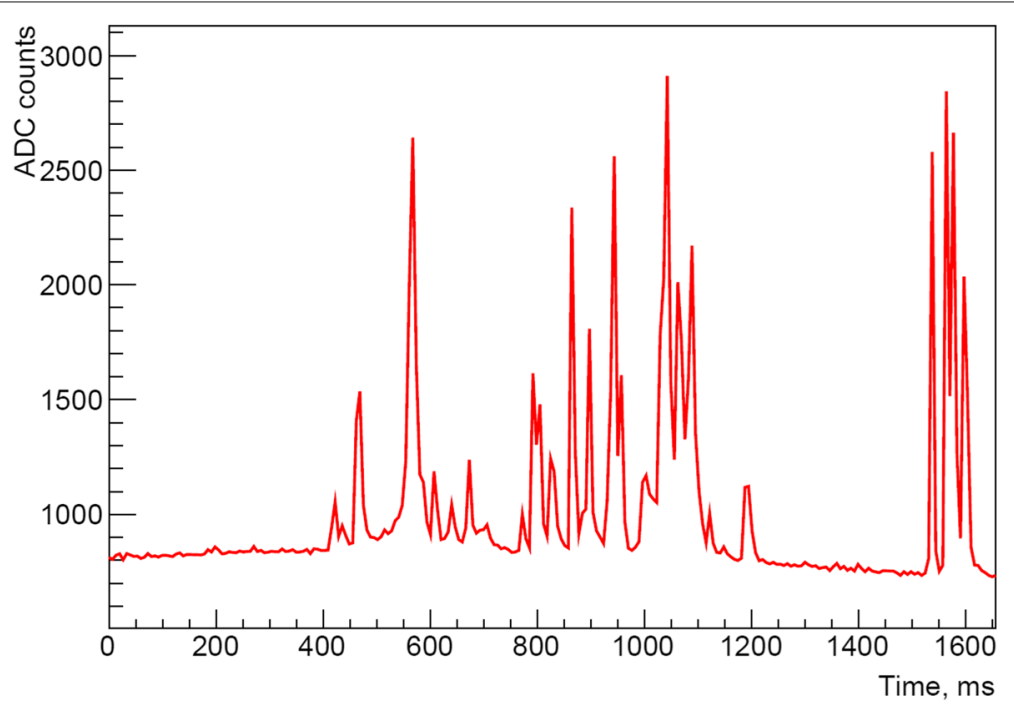

Fig. 4 Waveforms of a pixel brightness for an event detected at 03:23:27 UTC, January 4, 2017 above South America. Sampling time is equal to 6.6 ms 
axis. Simultaneous observations of TGFs in gamma, opti$\mathrm{cal}$, and radio bands are the main advantage of the TARANIS project (Farges et al. (2017)). For this purpose, the payload will include small aperture optical cameras, which provide TLE imaging in two spectral ranges of 757-767 and 772-782 nm that helps separate typical lightning and sprite effectively. Apart from two cameras, the TARANIS payload includes photometers for TLE timing in four wavelength bands of $170-260 \mathrm{~nm}, 332-342 \mathrm{~nm}, 757-$ $767 \mathrm{~nm}$, and 600-900 nm. Photometers have the same FOVs as optical cameras, and are able to produce trigger for all instruments on board. Payload also will include wide band radio frequency analyzer for electromagnetic signal waveform measurements in the frequency range from few kilohertz up to $37 \mathrm{MHz}$. Besides of the thunderstorm and lightning events identification, this instrument will be used also to study electromagnetic disturbances caused by electron precipitation, as well, as for the study of electromagnetic wave propagation in the ionosphere, and of the near-Earth space and of electromagnetic environment monitoring.

The ASIM (Atmosphere-Space Interactions Monitor) project (Neubert et al. (2006)) was launched on April 2, 2018 for the deployment on-board of the International Space Station (ISS), and on April 13 it was docked to COLUMBUS module of the ISS. It was developed for studies of the thunderstorm phenomena, of their possible influence on the upper atmosphere, and, especially, for the extensive study of TLEs and TGFs. The payload of ASIM includes X-and gamma-ray instrument MXGS and the optical instrument MMIA. The MXGS instrument (Budtz-Jorgensen et al. (2009)) consists of imaging $\mathrm{X}$-ray telescope for the energy range of $10-400 \mathrm{keV}$, with the position-sensitive detector (PSD) as $128 \times 128$ matrix. A gamma-ray detector for the energy range of $0.2-40 \mathrm{MeV}$ was also considered for use. X-ray telescope provides an angular resolution better than $1^{\circ}$ in the mode of orientation to a point-like source. The optical instruments (MMIA) consist of the nadir-looking two cameras and three photometers.

The multi-pixel $(1024 \times 1024)$ CCD matrices are used as detectors in optical cameras, providing the spatial resolution about $300-400 \mathrm{~m}$ on the ground for the $400 \mathrm{~km}$ ISS typical orbital altitude. Photometer sampling rate of $\sim 100 \mathrm{kHz}$ provides the time resolution about $10 \mu \mathrm{s}$. The MXGS instrument is planned to be operated continuously, except during the South Atlantic Anomaly (SAA) passage, and the MMIA should be operated only at the night side of the ISS orbit. Along with data recording in a monitor mode, the detailed data records should be made by triggers produced by both instruments. The generation of cross-trigger of payload complex is also foreseen. The instrument input windows are directed to the local nadir and toward the Earth limb.
The main goal of TRYAD (Terrestrial RaYs Analysis and Detection) experiment is multi-point observations in order to verify TGF beam models. The experiment is based on two identical $6 \mathrm{U}$ cubsats which in-orbit separation controls via aerodynamic differential drag. It is equipped with plastic scintillator coupled with silicon photomultiplier (SiPM). The crucial parameter of the experiment is an acquisition time accuracy which is provided by embedded GPS module.

\section{The specific problems of TGF study}

The problem of TGF origin is of specific interest. Discussing the future research on TGFs is necessary to note that the physical nature of their sources is not completely understood. The main unsolved problem with the TGF origin is the putative connection of TGFs with electromagnetic discharges of thunderstorms, and with TLEs. The solution of this problem is usually connected to the necessity of complex observations in the multi-frequency range of electromagnetic emission, from radio (tens of $\mathrm{kHz}$ ) to gamma-rays (tens of $\mathrm{MeV}$ ). It is expected that $\mathrm{X}$-ray and gamma-ray instruments will provide localization of TGF source with the accuracy of a few degrees. For this purpose, it seems optimal to use combination of the wide-field detectors that will provide photometry with the high sensitivity, and of the Compton, or coded mask telescopes with the PSD, that will allow localization of the detected signal source. Detection of X-rays gives information about altitude of TGF source, because the X-ray intensity depends on matter thickness above the generation point. Identification of TGF source with given thunderstorm area or even with lightning requires the onboard simultaneous registration of event in radio, optics, and gamma. Coordinated observations with ground lightning observation networks, such as WWLLN and Vaisala are also necessary. For this purpose, the accurate (no less than $\sim 1 \mu \mathrm{s}$ ) binding of detected event to the universal time (UT) should be provided by the satellite board.

In order to compare favorably with discussed above very impressive instruments of TARANIS and ASIM missions, it makes sense to expand the energy range, in which position resolution is possible, up to $1 \mathrm{MeV}$, or even more, taking into account hard energy spectrum of the gamma radiation from TGFs. It will be advantageous to combine the good space resolution capability with the possibility of high-resolution spectroscopic measurements in the range of up to $\sim 10 \mathrm{MeV}$ that will permit observations of TGFs in the gamma-ray nuclear lines. Taking into account, that TGFs are very short and the time resolution of the instrument under development has to be better than $\sim 10 \mu \mathrm{s}$. The complex pixelated detector, composed of a large number of small independent sensitive elements, has much smaller chances for pile-up effects, than the large single crystal detector. It is also desirable to have capability 
to detect electrons by the same instrument, which could be achieved by using X-and gamma-ray detectors for detection of electron bremsstrahlung.

The other approach, which could provide localization of TGF's source, is based on the use of triangulation technique for observations of the selected thunderstorm area from different satellites. Such method is very successful for cosmic gamma-ray burst (GRB) localization. In the case of TGFs it needs at least three satellites, which should be spaced at a distance of several hundred kilometers for orbits with altitude about $400-600 \mathrm{~km}$. In this case, the GPS time accuracy will be enough for determination of time delay of TGF signals detected by different satellites, which gives thunderstorm area localization in limits of tens of kilometers. The separate problem is necessary to prevent the spread of satellites on distances more than abovementioed several hundred kilometers to provide simultaneous observations of given area from all satellites.

According to (Babich and Roussel-Dupré 2007), a photo-nuclear reaction, ${ }^{14} N(\gamma, n){ }^{13} N$, may occur at TGF source or in surrounding matter because gamma-rays with energies above threshold of $10.5 \mathrm{MeV}$ have been actually observed. Thus, photonuclear neutrons may be the clue to solve the problem of non-thermal mechanism in thunderstorm activity. Neutron production in TGFs was studied by (Carlson et al. 2010), who predicted that TGF produces $\sim 10^{12}$ neutrons at an average, that corresponds to a ground-level neutron fluence of $(0.03-1) \times$ $10^{4} \mathrm{~m}^{-2}$. Similar predictions were made by (Babich et al. 2010), who obtained neutron fluence of $10^{3}-10^{7} \mathrm{~m}^{-2}$ at ground level from gamma rays produced under the RREA mechanism. According to (Babich et al. 2010) a yield rate of a photonuclear neutron per one gamma quantum with energy over $10 \mathrm{MeV}$ is $4.3 \times 10^{-3}$. An attenuation length of $20 \mathrm{MeV}$ neutrons in the atmosphere was calculated as $\lambda_{n}=13 \mathrm{~g} \mathrm{~cm}^{-2}$ with the use of cross section of elastic and/or inelastic collisions with air nuclei (Shibata 1994).

The simulation of neutrons generated by gamma quanta during their propagation in the atmosphere was made also by (Drozdov et al. 2010) and (Grigoriev et al. 2010). They simulated the albedo neutron background and thunderstorm-produced neutrons, and then compared albedo and thunderstorm neutrons spectral parameters to estimate possibility of thunderstorm neutron detection in orbits with different altitudes up to $450 \mathrm{~km}$. The energy spectrum of thunderstorm neutrons spreads from $\sim 1 \mathrm{keV}$ to $\sim 10 \mathrm{MeV}$, with a maximal flux at $\sim 6 \mathrm{MeV}$. Due to the scattering in the atmosphere, the main part of neutrons reaches orbital altitude from the point of origin in about several dozen of milliseconds. The maximal value of thunderstorm neutron integral flux will be observed in the time interval of 10 to $20 \mathrm{~ms}$ after the TGF, with the flux level up to $1.6 \mathrm{~cm}^{-2} \mathrm{~s}^{-1}$.
In view of the recently published results on the photonuclear processes and neutron generation during thunderstorms (Enoto et al. (2017)), the possibility of neutron detection in the range from $\sim 1 \mathrm{MeV}$ up to $\sim 20 \mathrm{MeV}$ will be also useful to have a direct indication of photo nuclear reactions in the TGF source. Thus, it is an independent channel, which allows estimation of hard energy photon number in the source. The other way is that neutron flux gives the estimation of accumulation of long-lived radionuclides in the atmosphere if they can be produced also in the gamma quantum interaction with the nuclei in the atmosphere.

All above considerations should be taken into account when choosing future advanced instruments for the TGF study. The present state of the field calls for the development of a new generation of gamma-ray imager/spectrometer with high resolution and sensitivity. Such instrument should be added by neutron detector or should be sensitive to the neutrons. It opens the way not only to multi-wavelength but also to a kind of multimessenger observations.

\section{Concept of TLE and TGF observations in the Universat-SOCRAT project}

The next step in TAE study could be connected with Universat-SOCRAT space project of Lomonosov Moscow State University (MSU). It is a multi-satellite project for real-time monitoring in the near-Earth space of potentially dangerous hazards, that means the radiation environment; dangerous objects of the natural (asteroids, meteors) and of technogenic origin (space debris), as well as electromagnetic transients, i.e., cosmic gamma-ray bursts, optical, ultraviolet, and gamma-ray flashes originating in the Earth's atmosphere. Study of TGFs and TLEs is one of the high priority goals of this project.

Within the framework of the Universat-SOCRAT project, a few small satellites should be placed into specially selected orbits. For the minimal version, the group of satellites could consist of three satellites (Panasyuk et al. 2015). One spacecraft of medium mass (small satellite) should be launched on a low solar-synchronous orbit with an altitude of about $500-650 \mathrm{~km}$ and an inclination of $97^{\circ}-98^{\circ}$. Two other satellites of lower mass (micro satellites) should be launched on an orbit close to circular with an altitude of about $1400-1500 \mathrm{~km}$ and an inclination of $\sim 80^{\circ}$ and on an elliptical orbit with an apogee of about $8000 \mathrm{~km}$, a perigee of about $600-700 \mathrm{~km}$, and an inclination of $63.4^{\circ}$. The mutual arrangement of the orbits is shown in Fig. 5.

The small satellite payload should include (Panasyuk et al. 2017) instruments for monitoring the space radiation, a set of instruments for optical monitoring of hazardous objects, a set of instruments to study atmospheric phenomena in the optical range, a set of instruments for 


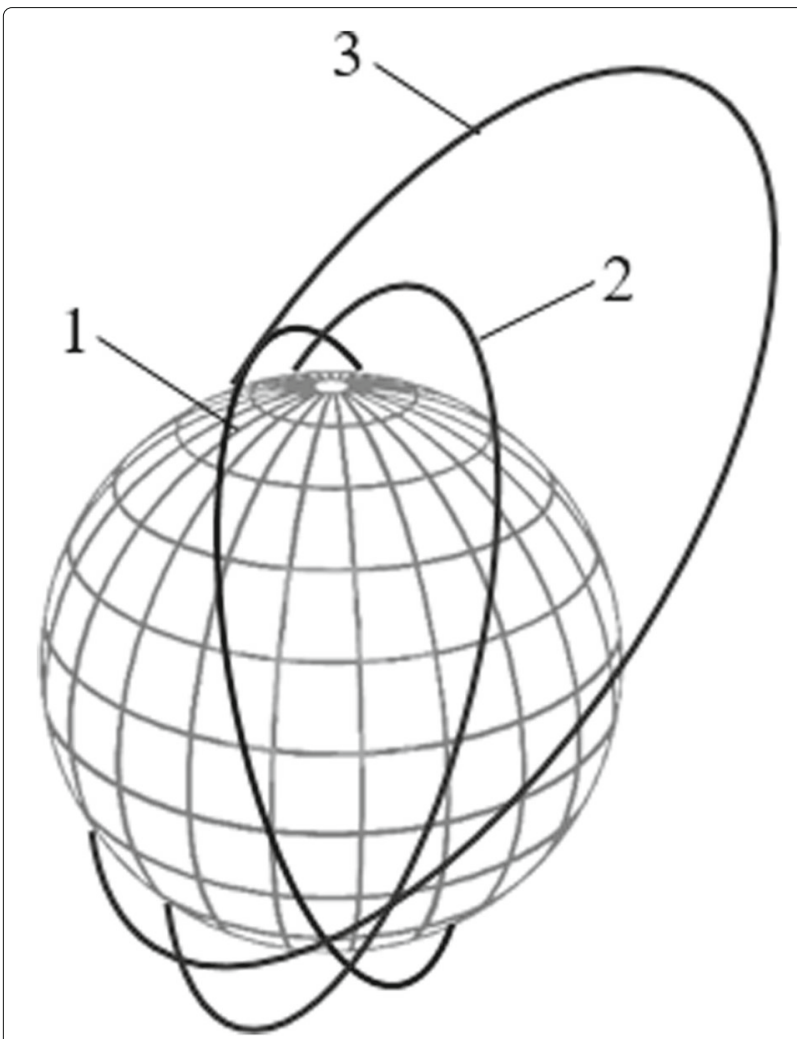

Fig. 5 The mutual arrangement of multi-satellite group orbits. The numbers mark the orbits in the order presented in the above text

monitoring in the gamma-ray range, and a special unit for data collection. The payload should also include threecomponent magnetometer. The payload of each micro satellite should include instruments for space radiation monitoring, a compact gamma spectrometer, a wide fieldof-view optical camera, an ultraviolet detector, and an electronics unit for data collection.

Study of TLEs and TGFs will be realized mainly on the low orbital small satellite. Its polar orbit is not optimal for observations in gamma rays due to variable background. However, it provides a good opportunity to observe the different parts of the Earth's atmosphere including both equatorial and polar regions that significantly expands the possibilities of experiment in search of new kinds of TAE-like phenomena. On the other hand, the background conditions near the equator are quite acceptable for TLE and TGF study. The main scientific objectives from this point are multi-wavelength and multi-messenger observations. The first means the complex study of TGFs and TLEs in wide bands from red optics to gamma. The multi-messenger observations mean detection of high-energy electrons and neutrons simultaneously with optical, UV, X-ray, and gamma photons. The direct electron detection from thunderstorm electron, gamma-ray avalanche, gives the direct confirmation of the theory of runaway electron breakdown and may confirm the mechanism of high-altitude discharges initiated by strong electric fields in the thunderstorm atmosphere. The neutron detection is necessary for estimations of hard photon number in TGF source as well as for accumulation of long-lived radionuclides in the atmosphere.

\section{Experimental methods of TLE and TGF observations on the main satellite of the Universat-SOCRAT multi-satellite group}

\section{Instruments for TLE studies}

The early MSU satellites provided a large amount of information on the properties of UV transient emission from the Earth's atmosphere, but at the same time they put forward a list of new puzzles that can be solved only by a new type of space-based telescope with spectrometric capabilities. Let us summarize these new problems that need to be studied, as well as the parameters of the detector needed to accomplish the task.

1 Long series of flashes have been detected. These series can be up to 10 min duration that corresponds to the distance of $4-5000 \mathrm{~km}$ along the satellite track. Series occur mostly above the regions of lightning activity but can continue even when the satellite leaves thunderstorm area. These flashes have various nature, but they point to a large-scale atmospheric activity.

2 Complicated temporal structure of UV flashes is observed at different time scales (from microseconds to seconds). But only short and separate time intervals were detected. Further studies with detectors having high spatial resolution are needed for better understanding of inter-relations of these events, as well, as continuous measurements with high-temporal resolution.

3 More detailed spectral information is needed to classify observed transients, understand their origin, and estimate more precisely altitude of their generation and development.

4 Energy distribution of the UV flashes detected in the previous experiments was calculated in the assumption of the nadir direction to the event. Absence of spatial resolution does not allow more accurate measurements of UV-flash energy. We note that good spatial resolution in addition to temporal and spectral resolutions of the used detectors is very important.

As a result of the considerations discussed above, a new detector mini-lens telescope-spectrometer) was developed for the Universat-SOCRAT project based on the 
previous experience in UV detectors manufacturing at SINP MSU. This detector is aimed for monitoring measurements of transient atmospheric events with a high $(\sim 1 \mu s)$ temporal resolution in a number of wavelength bands. MLT-S will provide imaging of the events, energy distribution in the spectrum of optical radiation (from near UV to NIR), and temporal profile of the signal.

The detector consists of three main parts:

1 Telescope;

2 Spectrometer;

3 Data processing system.

The 3D model of the detector is shown in Fig. 6. Dimensions of the detector are $20 \times 20 \times 30 \mathrm{~cm}$, total power consumption does not exceed $10 \mathrm{~W}$ and mass of the detector is near $7.2 \mathrm{~kg}$. The most massive part is an aluminum box, so the weight of the flight model of the MLT-S can be significantly decreased by using carbon-plastic materials in the mechanical structure. Entrance windows of the telescope and spectrometer are in the nadir direction and equipped with black blends which protect the detector from the side illumination.

The MLT-S telescope is based on the experience of miniEUSO development (Capel et al. 2018) and represents a detector with a simplest optical system composed of one lens of $5 \mathrm{~cm}$ diameter and a photodetector. The lens has a spherical front side (radius of curvature is equal to $82.32 \mathrm{~mm}$ ) and plane rare side. This lens provides 50 times more sensitivity than detectors on satellites Tatiana and Vernov (UV flashes with number of photons released in the atmosphere $\sim 10^{15}$ can be measured).

The photodetector is one elementary cell (EC) of the mini-EUSO detector and consists of four multi-anode photomultiplier tubes (MAPMTs) which are placed in a focal plane of the lens. Total amount of pixels on the focal surface is 256 which compose a matrix $16 \times 16$. EC has one Cockroft-Walton high-voltage (HV) power supply, which provides HV for all MAPMTs and is controlled by the data processing system in order to change the gain of the MAPMTs for the cases of high brightness of the event or high level of UV background.

Each pixel of MAPMT has $\sim 3 \mathrm{~mm}$ size, and focal length of the optical system is $15 \mathrm{~cm}$. For this configuration, the telescope resolution is $1.2^{\circ}$, which provides a spatial resolution on the Earth's surface $\sim 10 \mathrm{~km}$ for $500 \mathrm{~km}$ altitude of the satellite orbit. These parameters lead to observed area of $25,600 \mathrm{~km}^{2}$ in the atmosphere. The optical scheme and ray tracing for $0^{\circ}, 4^{\circ}, 8^{\circ}, 9^{\circ}$ zenith angles are shown in Fig. 7.

The MAPMT is operated in the single-photoelectron mode and provides high sensitivity of the detector. This mode is performed by using an application-specific integrated circuit (ASIC) of the SPACIROC-3 (Spatial Photomultiplier Array Counting and Integrating Read Out Chip) type, which was developed earlier for the JEMEUSO experiment (Blin-Bondil et al. (2014)). Photons are counted by SPACIROC-3 for 1 or $2.5 \mu$ s time bins that correspond to temporal resolution of the detector.

Spectrometer is made of 16 single-anode PMTs Hamamatsu R1463 which were used in the TUS experiment (Klimov et al. (2017)) on-board the Lomonosov satellite (Sadovnichii et al. (2017)). These PMTs compose a matrix $4 \times 4$ and each PMT has an individual filter on the entrance window. It means that each PMT measures the radiation from the whole FOV, but in a specified wavelength range.

We hope that measurement of the transients spectrum will allow us to determine the type and altitude of the

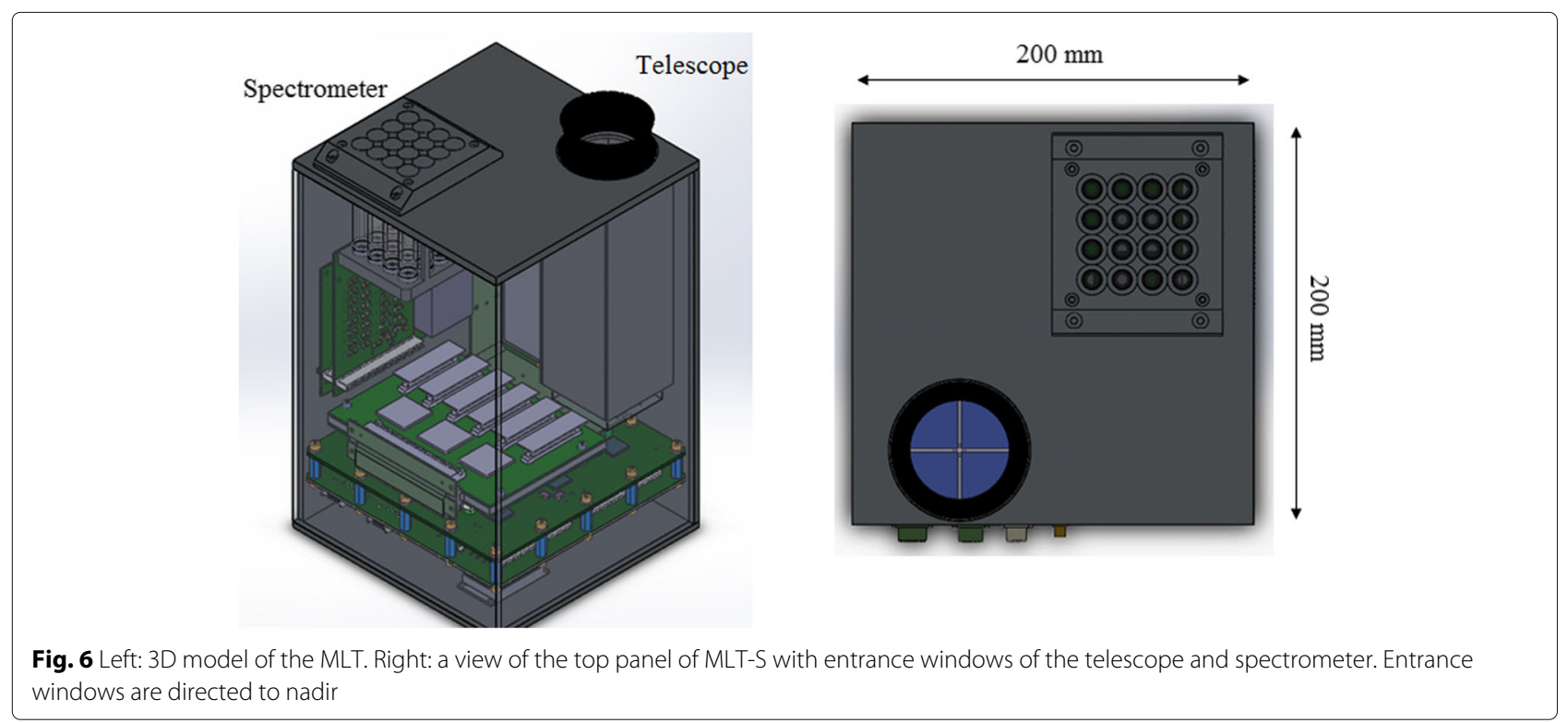




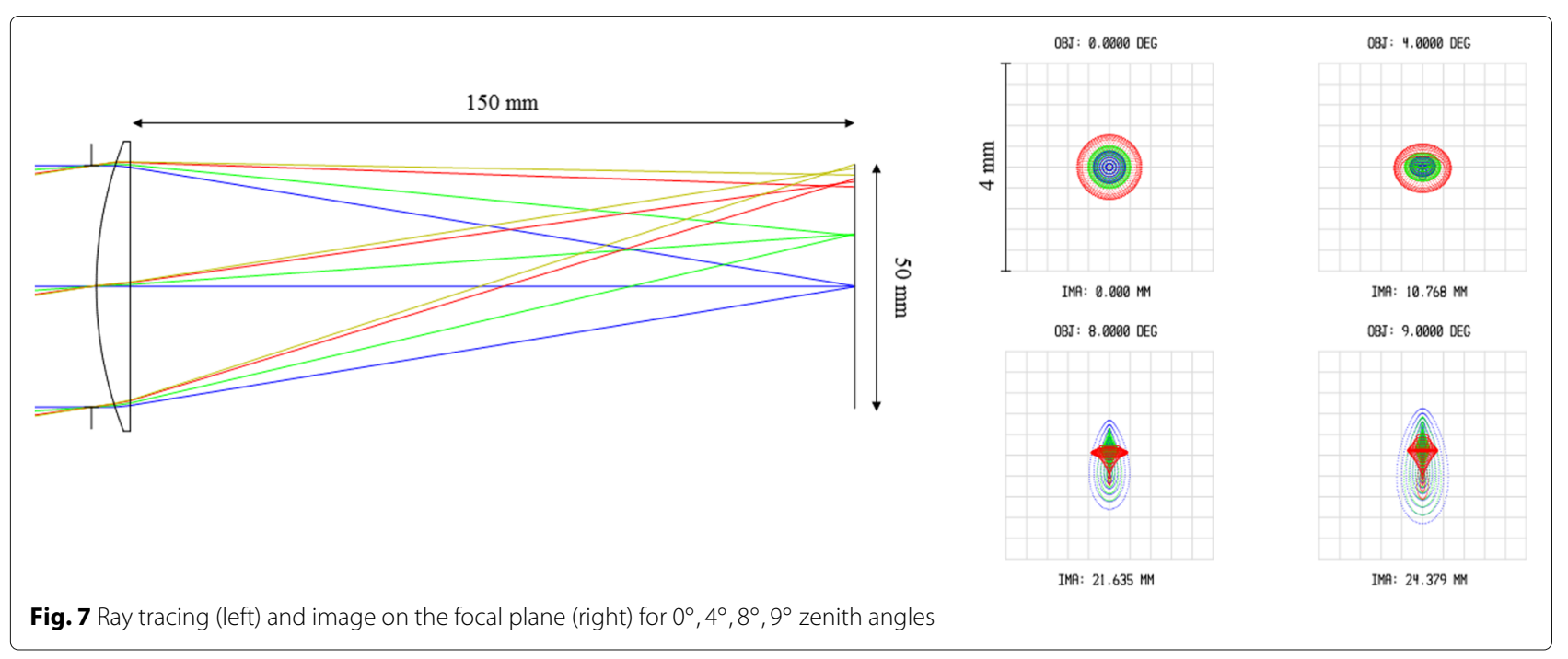

event. UV and NIR filters will be used to have a possibility to compare new results with that of the previous MSU experiments (Tatiana-1, Tatiana-2, and Vernov), where UFS1 (240-400 nm) and KS11 (600-800 nm) filters were used. This will allow us to make preliminary estimates of the event height using the old method, suggested by (Garipov et al. 2013) and applied to Tatiana-2 data.

We plan to use the atomic oxygen absorption line $(762 \mathrm{~nm})$ additionally in order to separate low-altitude events from the high-altitude atmospheric luminous phenomena. The chosen oxygen absorption line should be faint for lightning discharges occurring deep in the atmosphere. The same spectral bands were used by spectrophotometers on-board ISUAL (Chern et al. (2003)) and JEM-GLIMS (Ushio et al. (2011)). On the other hand, one of the most intense lines in the lightning spectrum at $777 \mathrm{~nm}$ (Orville and Henderson 1984; Sharkov et al. 2018) will be used for detection of lightning events. Other spectral bands can be selected for various purposes, like to derive an altitude of the event or mean energy of electrons in the discharge, etc. All spectral bands suggested for use in the spectrometer are summarized in Table 1.

In comparison with already performed missions (ISUAL, JEM-GLIMS) and other planned missions, like ASIM and TARANIS, a number of spectrophotometers in a new MSU spectrometer is enlarged to 16 , comparing to previously used 4 or 6 , and the selected temporal resolution is much better, i.e., $\sim 1 \mu \mathrm{s}$ vs $50 \mu$ s of JEM-GLIMS, for example. This fact will allow measurements of the temporal and spectral structure of the atmospheric events in much more details, as well as to achieve a reliable separation of various types of atmospheric phenomena.

Data processing system of the spectrometer will be based on a Xilinx Zynq XC7Z030 system on chip. This chip includes both a high-speed logic part (a field programmable gate array-FPGA) and a processor
Table 1 Spectrometer wavelength bands

\begin{tabular}{|c|c|c|}
\hline \# & Wavelength $(\mathrm{nm})$ & Comment \\
\hline 1. & $240-400$ & $\begin{array}{l}\text { UFS1, UFS2, KS11 filters for comparison with } \\
\text { MSU experiments (Tatiana-1, Tataina-2, and } \\
\text { Vernov) }\end{array}$ \\
\hline 2. & $270-380$ & \\
\hline 3. & $>600$ & \\
\hline 4. & 762.7 & TLE marker-atomic oxygen absorption line \\
\hline 5. & 777.4 & $\begin{array}{l}\text { Lightning marker - the brightest line in } \\
\text { lightning spectrum, used for lightning } \\
\text { detection in LIS ( (Orville and Henderson } \\
\text { 1984)) and convergence ((Sharkov et al. } \\
\text { 2018)) experiments }\end{array}$ \\
\hline 6. & 500.1 & $\begin{array}{l}\text { Lightning marker-bright line in lightning } \\
\text { spectrum and absent in sprite spectrum } \\
\text { ((Milikh et al. 1998)) }\end{array}$ \\
\hline 7. & 337 & $\begin{array}{l}\mathrm{N}_{2} 2 \mathrm{P} \text { line-marker of low latitude and } \\
\text { should absent in lightning spectrum due to } \\
\text { ISUAL data }\end{array}$ \\
\hline 8. & 391.4 & $\mathrm{~N}_{2}^{+} 1 \mathrm{~N}$-line of ionized atomic nitrogen \\
\hline 9. & 427.8 & $\begin{array}{l}\mathrm{N}_{2}^{+} 1 \mathrm{~N} \text { present in aurora light and marker of } \\
\text { ionization source (energetic charge particle } \\
\text { flow) }\end{array}$ \\
\hline 10. & 470.9 & \\
\hline 11. & 690 & $\begin{array}{l}\text { Meinel band-marker of high latitude and } \\
\text { high energy of electrons involved into the } \\
\text { process }\end{array}$ \\
\hline 12. & 388.3 & $\begin{array}{l}\text { Cyanogen (CN) emission, a spectral signature } \\
\text { of lightning flashes with continuing current } \\
\text { (monitoring of lightning that can cause } \\
\text { forest fires ((Christian et al. 1983; Fuquay et al. } \\
\text { 1967)). }\end{array}$ \\
\hline 13. & 294 & $\begin{array}{l}\text { Signature of extremely high altitude event } \\
\text { (lines of } \mathrm{N}_{2}(\mathrm{VK}) \text { system) }\end{array}$ \\
\hline 14. & 313 & \\
\hline 15. & 399.8 & $\begin{array}{l}\mathrm{N}_{2} 2 \mathrm{P}(1,4) \text { analyzed by (Armstrong et al. } \\
2000 \text { ), in comparison to } 427.8 \mathrm{~nm} \text { and } \\
470.9 \mathrm{~nm} \text { lines }\end{array}$ \\
\hline 16. & Without filter & $\begin{array}{l}\text { Total emission of lightning or TLE } \\
\text { measurements }\end{array}$ \\
\hline
\end{tabular}


system. It allows development of a high-speed dataprocessing system and implementation of different triggering algorithms. Using of 1 Gb DDR memory provides continuous writing of information with high frequency directly from the FPGA part via high-speed Ethernet interface to the board computer to store all this information.

The whole system can operate in different modes with different triggers. The first mode is a continuous monitoring of the atmospheric emission with high temporal resolution of $2.5 \mu \mathrm{s}$. This mode allows measurements of UV emission along the satellite track almost without dead time, and thus will provide information on the transient temporal structure with high resolution. It is necessary in order to understand the series of flashes detected in previous MSU experiments. This mode will produce a huge amount of data; therefore, only a few channels of the spectrometer will be used during these measurements and on the selected part of the orbit. Slow monitoring mode with temporal resolution of $\sim 1 \mathrm{~ms}$ (similar to one used during the Tatiana and the Vernov experiments) is selected as the second mode. This mode produces three orders of magnitude less information in comparison to the first one, and thus will allow simultaneous measurements by telescope and spectrometer. This mode will give us information on spatial structure of events in series. The third mode of spectrometer is a variety of triggering regimes, like (1) several threshold triggers of spectrometer with various temporal resolutions, $2.5 \mu \mathrm{s}, 0.2 \mathrm{~ms}$, and $3 \mathrm{~ms}$, which select events exceeding a threshold in a definite spectrometer channel, and (2) a number of telescope triggers in which a spatial structure of the events is analyzed in addition to amplitude. It will allow selection of the events with a specific geometry (such as elves, for example, where a ring structure is expected, or, alternatively, a linear track of meteors).

In addition to MLT-S, the satellite will be equipped with a modified detector DUV similar to the detector used in the Vernov and Tatiana missions, but with an additional far UV channel (100-300 nm). This wavelength range is very useful for selection of high altitude events, because the emission from low altitude lightning in this? range is efficiently absorbed by the ozone layer. This way of TLE selection is partially used in the JEM-GLIMS spectrometer (Sato et al. (2017)).

\section{TGF observations in the Universat-SOCRAT project}

Instruments for TGF study of the Universat-SOCRAT project will include gamma-ray flash monitor (GFM), and a tracking gamma-ray spectrometer (TGS) of the high resolution and sensitivity, that will be sensitive also to neutrons with energies from about $1 \mathrm{MeV}$ up to several dozen of megaelectron volt. The separate unit for data analysis and control of GFM and TGS should be also foreseen in the payload. This unit should contain digital electronics unit that will provide the record of data stream with a time resolution of $\sim 10 \mu \mathrm{s}$, and generate the triggers enabling the detection of different type of events, including TGFs and neutron flux increases. The data record must be referenced to the UTC time with an accuracy of $\sim 1 \mu$ s to allow the positioning of the TGFs, if observed simultaneously by several space missions, using triangulation, and for the comparison with the data of ground based lightning location networks. Both instruments, TGS and GFM, should be installed on the medium mass satellite similar to that used for Vernov mission. It provides the payload mass about $100 \mathrm{~kg}$ that is enough for these instruments.

\section{Tracking gamma-ray spectrometer}

Tracking gamma spectrometer of high resolution and sensitivity is a combination of two space (position) sensitive units, so-called hodoscopic and calorimetric. Both units should be connected in a mono-block construction (see Fig. 8). The coded mask, with a random pattern coding aperture, will be placed above the calorimeter unit at a distance of $\sim 50 \mathrm{~cm}$. The instrument axis should be directed along the nadir-zenith axis-in such a way that the instrument should be directed to the local nadir from the hodoscope side, which should be used mainly for detection of neutrons from the atmosphere. In the case of TGF detection, such orientation allows estimation of detected quanta inclination angle using scattering parameters of those quanta that will deposit some part of their energy in at least two points of hodoscopic and calorimetric parts of the instrument. It also will allow effective separation of gamma quanta from neutrons and charged particles. Absorption of gamma quanta in the calorimetric unit also allows determination of their energy.

The combination of hodoscopic and calorimetric units assumes operation of the instrument in the mode of the dual Compton scattering. It permits to check the appearance of the GRB from the Earth's atmosphere and to separate real gamma-ray events as TGFs from the cases of electron precipitation. Image reconstruction using the coding mask telescope will allow localizing the GRB source and thus will help to separate GRBs from TGFs. The multi-pixel detectors used both in hodoscopic and in calorimetric units permit to eliminate significantly the contribution of pile-up pulses that is very important for detection of very fast events such as TGFs. The detection of false bursts imitated by cosmic ray energetic particles can be completely excluded with the use of multiple coincidences.

The instrument apart detectors will contain the data processing unit based on digital electronics that allows for very fast data recording with sufficiently high time resolution of $\sim 1 \mu \mathrm{s}$. It also should provide the on-board analysis of images obtained from the coding mask telescope and 

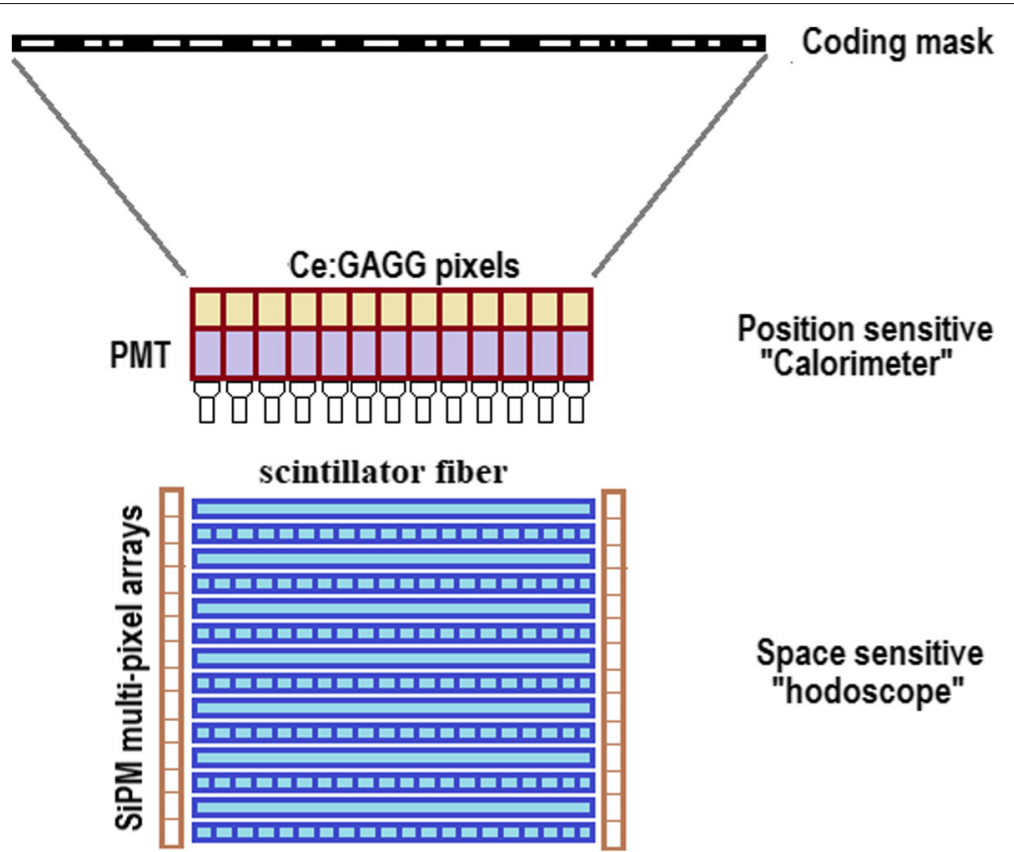

Fig. 8 The design of tracking gamma spectrometer

from the system of hodoscope-calorimeter-operated in the double Compton scattering mode, as well, as to generate the GRB and TGF triggers.

PSD in hodoscopic and calorimetric units allows localization of the place of particle or quantum interaction in the detector. It makes possible to obtain information about space distribution of detected fluxes. To the present, the main directions of PSD development for gamma-ray astronomy are connected to improving the PSD detection efficiency, to lower the detection threshold, to increase the accuracy of the source localization, to reduce the distorting effect of the "dead time" of the detector, and to improve the time resolution.

The both calorimetric and hodoscopic units of the instrument should be consisted of a number of identical modules, providing possibility for increasing of the effective area and thickness of the detector, depending on the overall mass resources of spacecraft. Then, we will consider the instrument parameters for calorimetric and hodoscopic units in a minimally reasonable configuration.

Thus, the hodoscopic unit of tracking gamma spectrometer should be a set of light-conducting scintillation fibers of small thickness $(0.15 \mathrm{~cm}$ or smaller), located in layers in such a way that in adjacent layers, the orientation of the fibers is normal to each other (see Fig. 8). This system allows separation of events connected to the detection of gamma quanta and neutrons. The principle of detecting gamma quanta is determined by their Compton interactions in fibers, and the detection of fast neutrons occurs in the mode of their elastic scattering by hydrogen nuclei in the scintillation fiber (Iyudin et al. 2015). The low-fiber thickness provides a high-spatial resolution when gamma quanta and neutrons will be detected, as well as a low threshold for the detected photon under the condition that the energy must be released in the interaction act at least in two neighboring layers. The multi-pixel matrices of SiPMs will be used as photosensors for recording light flashes in fibers. The matrix photosensitive surfaces should be facing the ends of fibers, positioned in such a way that each fiber is viewed by the corresponding cells ("pixels") of two photosensors (SiPMs).

We are currently planning to use $12 \times 12$ quadratic matrices of SiPMs, with a $3 \times 3 \mathrm{~mm}$ cell size of SenSL production. The dimensions of one matrix are $48 \times 47 \times$ $10 \mathrm{~mm}^{3}$, and its mass is $26 \mathrm{~g}$. Thus, for the minimal configuration with a hodoscope size of $150 \times 150 \times 150 \mathrm{~mm}^{3}$, it is necessary to use $36 \mathrm{SiPM}$ matrices of $12 \times 12$ dimension to read-out light pulses from the hodoscope.

An advantage of this instrument is the possibility of implementing different triggers. One of them will allow selection of the events corresponding to the elastic scattering of fast neutrons with energies from 5 to $100 \mathrm{MeV}$, based on the kinematics of the scattering event itself in the presence of two (or more) neutron interaction points in the volume of the hodoscope. While under conditions of an increased background of charged particles, it is possible to use a trigger where outer layers of the hodoscope are used for the purpose of protecting the internal part of the instrument from the charged particle penetration into it. Thus, the anti-coincidence shield is realized. The trigger 
condition can be checked by the digital unit according to the given algorithm, and it is possible to correct the algorithm, depending on the specific background conditions and the choice of the most priority tasks.

The calorimetric unit will consist of a set of minimum 1616 scintillating crystals of Ce:GAGG type, with size of $1.0 \times 1.0 \times 3.0 \mathrm{~cm}^{3}$ each, that should be placed on the top of the instrument. In the case of detection of TGF, calorimetric unit will detect gamma quanta scattered in the hodoscope. Ce:GAGG crystals have quite high light output $(\sim 57000$ phot $/ \mathrm{MeV})$ and density $\left(\sim 6.7 \mathrm{~g} / \mathrm{cm}^{3}\right)$ with sufficiently small decay time of $\sim 80$ ns (Iwanowska et al. (2013)), that ensures a high throughput of the unit. The fast crystals help to realize the high accuracy of the time measurements and high throughput of the measuring channel. Relatively high density of Ce:GAGG crystals provides the high efficiency of gamma quantum detection in wide energy range. Due to the high light output Ce:GAGG scintillators also give good energy resolution. For example, we have tried Ce:GAGG pixels with thickness of $3.0 \mathrm{~cm}$ and found the high-detection efficiency of gamma quanta with energies up to a several $\mathrm{MeV}$, and an energy resolution of $\sim 4 \%$ at $1 \mathrm{MeV}$.

The SiPM light sensors or small vacuum photomultipliers could be used for viewing of Ce:GAGG crystals. For example, Hamamatsu R1463 is quite compact photomultiplier with a diameter of input window of $13 \mathrm{~mm}$. R1463 photocathode is sensitive at the wavelengths of $300-650 \mathrm{~nm}$, with maximum cathode sensitivity at about $420 \mathrm{~nm}$, that is in good correspondence with the emission spectrum of Ce:GAGG.

The tracking gamma spectrometer has its own read-out unit of analogous and digital electronics.

The instrument parameters are given in the Table 2.

Experimental test of energy resolution of a one-pixel element of the calorimeter was done with a scintillating detector module based on the mirror-coated Ce:GAGG crystal with the size of $10 \times 10 \times 10 \mathrm{~mm}^{3}$ attached to the PMT of Hamamatsu R1463 type. A standard set of radioactive gamma-ray sources including ${ }^{137} \mathrm{Cs},{ }^{207} \mathrm{Bi}$, and ${ }^{241} \mathrm{Am}$ were used for module calibration. Experimental measurements of energy resolution are useful because several factors including non-uniformity of light collection in the detecting crystal influence on it.

The value of energy resolution was determined from the full width at the half maximum of the corresponding peak on the energy spectrum. The minimal and maximal energy of the range also were determined. The low energy threshold was $\sim 20 \mathrm{keV}$, and the upper limit for the measurements was $\sim 10 \mathrm{MeV}$. Low limit of the detector energy range is mostly determined by the absorption of soft gamma-rays in the substance of the instrument input window while the maximal value is determined by electronics. It was confirmed by the measurements that Ce:GAGG
Table $\mathbf{2}$ The tracking gamma spectrometer parameters

\begin{tabular}{ll}
\hline Parameter, units & Value \\
\hline Energy range for gamma quanta, MeV & \\
All interactions & $0.02-10.0$ \\
Coding mask telescope & $0.02-1.0$ \\
Double Compton imaging & $0.5-10.0$ \\
Effective area, $\mathrm{cm}^{2}$ (all interactions) & $\sim 250$ \\
Angular resolution & \\
Coding mask telescope & $\sim 2^{\circ}$ \\
Double Compton imaging (at 1 MeV) & $10-15^{\circ}$ \\
Time resolution, ns & 5 \\
Energy resolution (at 1 MeV) & $5 \%$ \\
Sensitivity to the 1 ms burst detection, $\mathrm{cm}^{-2}$ & $3 \cdot 10^{-2}$ \\
Energy range for neutrons, MeV & $3-100$ \\
Effective area for neutrons, $\mathrm{cm}^{2}$ (at $40 \mathrm{MeV}$ ) & 13 \\
Mass, kg & 40 \\
Information capacity, MB/day & $\sim 500$ \\
Power consumption, W & 60 \\
\hline
\end{tabular}

scintillator has stop-factor high enough for good resolution of $1.17 \mathrm{MeV}$ and $1.33 \mathrm{MeV}$ lines from ${ }^{60} \mathrm{Co}$, even using quite small crystal with the size of $10 \times 10 \times 10 \mathrm{~mm}^{3}$.

The energy resolution at $1.77 \mathrm{MeV}$ (isotope ${ }^{207} \mathrm{Bi}$ ) is $4.6 \%$. The energy dependence of the resolution of the detector with Ce:GAGG can be approximated by inverse proportionality to the root of the energy so the main contribution to the energy resolution is due to fluctuations in the number of photo-electrons, and the expected effect of non-uniform light collection is not significant.

Measurements of the light absorption in the scintillation fiber were also made in order to test the possibility to use such fiber as an element of the hodoscope in correspondent energy range. The measurements were made for two kinds of fibers with square section and the size of $3 \mathrm{~mm}$ and $1.5 \mathrm{~mm}$. In each measurement, several layers of fibers were placed into a special grid. The light-sensitive SiPM array was placed at the end face of the fibers. For the measurements, a collimated source of the ${ }^{90} \mathrm{Sr}$ decay electrons with a $\sim 5 \mathrm{~mm}$ diameter beam was used. The source was placed above the fiber in such a way that the radiation spread along one column of the multi-pixel photodetector. The coincident events in at least two upper layers of fibers were selected, in order to guarantee that the energy release in the layer nearest to the source of electrons was $\sim 600 \mathrm{keV}$ for $3 \mathrm{~mm}$ fiber and $\sim 300 \mathrm{keV}$ for $1.5 \mathrm{~mm}$ fiber. The source moved along the fiber, the amplitude spectra were collected for each position, and the channel of the peak maximum for the collected amplitude spectrum was determined. The measurements show that $15 \mathrm{~cm}$ long fiber with $1.5 \mathrm{~mm}$ quadratic section absorbs 
not more than $50 \%$ of the scintillation light trapped by the fiber. More light is collected when the light is collected from the both ends of the fiber that permits us to conclude that such fibers can be successfully used in the TGS hodoscope.

We have tested also the time resolution for different combinations of scintillating crystals and of photosensors. The test was done with the use of a coincidence method, using radioactive sources producing two gamma quanta at the same time. Isotope ${ }^{22} \mathrm{Na}$ emitting two annihilation gammas with energy $511 \mathrm{keV}$ in opposite direction was selected, as well as ${ }^{60} \mathrm{Co}$ that decays producing two photons with energies of $1.17 \mathrm{MeV}$ and $1.33 \mathrm{MeV}$, with the picosecond delay. The decay events with pulses produced in two detectors were used as a start and stop signals that were fed into the board of time-code converter P7889, by FAST ComTec company, providing the measurement of time delay spectra with 100 picosecond resolution. Collecting and processing of time delay spectra was done by special MCDWIN software provided with the measurement board. The tests were made for the PMT+Ce:GAGG modules as well as for the SiPM+scintillating fiber modules, and for their combination. The time resolution of less than $1 \mathrm{~ns}$ was obtained in all combinations. This good timing will allow analyzing of the events produced by the gammas and neutrons in TGS at the distances of $\sim 10 \mathrm{~cm}$.

\section{Gamma-ray flash monitor}

The GFM instrument should provide the monitor type observations of the upper atmosphere in the hard X-rays and soft gamma-rays, in an energy range of $0.01-3.0 \mathrm{MeV}$, with the possibility of rough localization of the TGF source. GFM will consist of three identical detector units, which are similar to the detector unit used as gammaray burst monitor of the Lomonosov mission (Svertilov et al. 2018). This detector is also the successor of detectors that successfully registered TGF on the Vernov satellite (Bogomolov et al. (2017)).

The axes of GFM detectors should be oriented at $90^{\circ}$ relative to each other, similar to the mutually normal edges of the cube, thus forming a Cartesian coordinate system. With this configuration, the main diagonal of the cube will be directed to the local nadir. The possible location of GFM instrument on the satellite payload panel is shown in Fig. 9. The sensitive area of each detector has a cosine angular dependence which within $\sim 60^{\circ}$ of its axis is not shadowed by any element of the spacecraft (see Fig. 9). This configuration will allow the rough estimation of TGF source location in the atmosphere by comparison of the amplitudes of the different detectors output signal, similar to the KONUS concept of the GRB source localization (Mazets and Golenetskii (1981)).
Each detector unit consists of a layer of thin $(0.3 \mathrm{~cm})$ $\mathrm{NaI}(\mathrm{Tl})$ crystals positioned above a considerably thicker $(1.7 \mathrm{~cm})$ layer of $C s I(T l)$ crystals (see Fig. 10). The diameter of each of these scintillators is $13 \mathrm{~cm}$. The $\mathrm{NaI}(\mathrm{Tl})$ is placed on top of the $C s I(T l)$ crystal and optically coupled with it. Both scintillator layers are viewed simultaneously by a single Hamamatsu R877 PMT. The thickness of the $\mathrm{NaI}(\mathrm{Tl})$ layer is optimized to detect the soft photons. Energy ranges are $0.01-0.5 \mathrm{MeV}$ for the $\mathrm{NaI}(\mathrm{Tl})$ crystal and $0.05-3 \mathrm{MeV}$ for the $\mathrm{CsI}(\mathrm{Tl})$ crystal. In this configuration, the $\mathrm{NaI}(\mathrm{Tl})$ layer serves as the main detector for hard $\mathrm{X}$-ray timing, while the $C s I(T l)$ crystal is used as an active shield against background gammas, but it can be used also to detect gammas with energies up to few $\mathrm{MeV}$.

The GFM detector units' parameters are given in the Table 3.

The difference of decay times for the $\mathrm{NaI}(T l)(\sim 0.25 \mu \mathrm{s})$ and $C s I(T l)(\sim 2.0 \mu \mathrm{s})$ crystals provides an opportunity to separate light flashes in these crystals by the pulse shapes with the help of special electronics. A PMT signal with negative polarity is characterized by fast increase in amplitude that is followed by a slower exponential decay. The signals from a set of crystals integrated during the first $\sim 800$ ns after trigger are called "fast" component, during the following $\sim 3 \mu \mathrm{s}-$ the slow component. The ratio of fast to slow components will allow separation of the cases of energy deposition in different parts of the detector. It is necessary to note that due to sufficiently long decay time of $C s I(T l)$ crystal, the so-called pile-up effect can be significant in $N a I(T l) / C s I(T l)$ phoswich detectors. The BDRG Lomonosov experience indicates on that pileup effect is real at high counting rates. The only way to avoid pile-up is using of more fast scintillators. Thus, for example, possibility of using of $\mathrm{LaBr}_{3}(\mathrm{Ce})$ or $\mathrm{CeBr}_{3}$ and BGO combination except $N a I(T l) / C s I(T l)$ should be considered.

Detector response matrix was simulated with the use of MEGAlib toolkit v.2.29.01, which utilizes the GEANT4 databases (geant4-10-00-patch-03 (Zoglauer 2005; Zoglauer et al. 2006)). Several dozen of simulation sessions of gamma quantum and electron parallel beam interactions with the GFM detectors were made for various beam directions relative to the detector axis. These beam directions were characterized by zenith angle $\theta_{1}$ (i.e., the angle between beam direction and detector axis) and the azimuth angle $\theta_{2}$ (i.e., between the beam projection on the detector plane and the detector axis). In different sessions, the gamma-ray energy spectrum was chosen as a power law with the different power index values, and the band models were used also, with the different sets of $E_{c}, \alpha, \beta$ parameter values.

Our simulations determined the detector energy threshold to be about $20 \mathrm{keV}$ at $\sim 20 \%$ efficiency for $\mathrm{NaI}(\mathrm{Tl})$. Radiation with the energy values between 10 and $20 \mathrm{keV}$ 


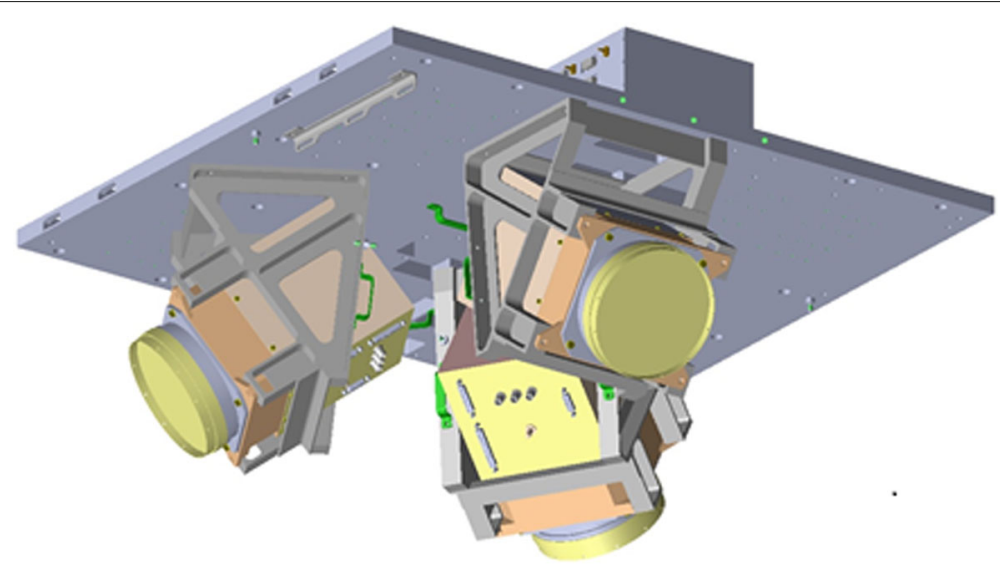

Fig. 9 The general view of the location of the GFM detector units

can be detected also but with the smaller efficiency of $\sim 5$ to $20 \%$. Gamma-rays having energy more than $200 \mathrm{keV}$ can pass through $\mathrm{NaI}(\mathrm{Tl})$ layer and penetrate the $C s I(T l)$ crystal. The efficiency of $C s I(T l)$ detector at $1 \mathrm{MeV}$ is about $30 \%$. Similar calculations were made for the electron beams with power energy spectra. The obtained energy threshold for electrons was derived as $\sim 300 \mathrm{keV}$ for $\mathrm{NaI}(\mathrm{Tl})$ and $\sim 2 \mathrm{MeV}$ for $\mathrm{CsI}(T l)$ crystals, respectively. Both values indicate the primary energy of electrons without energy losses in the instrument cover.

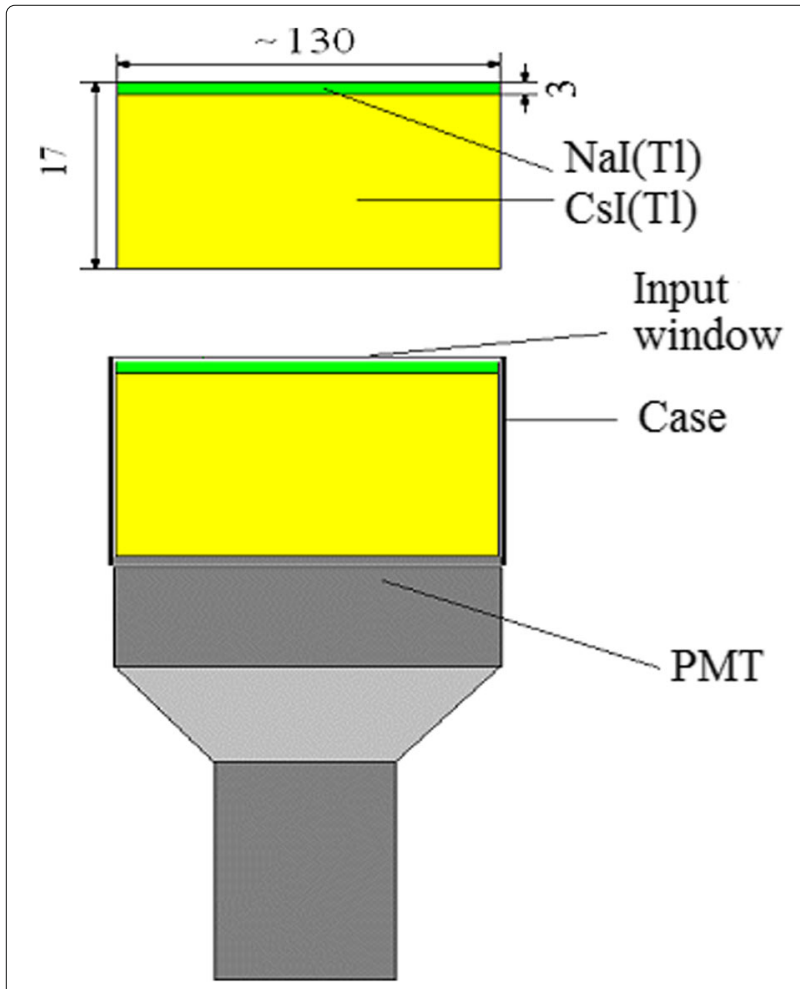

Fig. 10 Design of GFM scintillation detector
The phoswich method based on the combination of two-layer scintillator with different decay time such as $N a I(T l)$ and $C s I(T l)$ crystals viewed by a given PMT permits the effective separation of events in these scintillators. While observing certain burst-like count increase one can compare numbers of events with energy release in the $\mathrm{NaI}(\mathrm{Tl})$ and $\mathrm{CsI}(\mathrm{Tl})$ crystals. Gamma quanta with energies of about several hundred kiloelectron volts will interact both in $\mathrm{NaI}(\mathrm{Tl})$ and $\mathrm{CsI}(\mathrm{Tl})$ scintillators. In the case of electrons with similar primary energies, events with energy released only in $C s I(T l)$ will be absent. This approach is sufficient for effective discrimination of the real gamma-bursts against the background of electron flux variations, including short, precipitation-like, increasing intensity phenomena.

The TGF source location can be estimated by comparing the output readings $N_{i}$ of the three GFM detectors using the cosine function to adjust the beam coordinates. The axes of the detectors are shifted at $90^{\circ}$ from each other so that the TGF source angle coordinates $\theta_{1}, \theta_{2}$, and $\theta_{3}$ can be calculated from the simple formulas:

$$
\begin{aligned}
& \cos \theta_{1}=\frac{N}{\sqrt{N_{1}^{2}+N_{2}^{2}+N_{3}^{2}}}, \\
& \cos \theta_{2}=\frac{N}{\sqrt{N_{1}^{2}+N_{2}^{2}+N_{3}^{2}}}, \\
& \cos \theta_{3}=\frac{N}{\sqrt{N_{1}^{2}+N_{2}^{2}+N_{3}^{2}}} .
\end{aligned}
$$

Used in the formula, $N_{i}$ values can be estimated as excess counts in a given detector if one compares detected gamma quanta during flash with the expected number of background counts, extrapolated from this detector's readings immediately prior to the burst beginning. Obviously, the TGF source position accuracy depends on the TGF fluence and the background count rate at the time of 
Table 3 GFM detector units' parameters

\begin{tabular}{ll}
\hline Parameter, units & Value \\
\hline Energy range, MeV & $0.01-3.0$ \\
Effective area (for three detectors), $\mathrm{cm}^{2}$ & $\sim 360$ \\
Time resolution, $\mu \mathrm{s}$ & 1 \\
Field of view, sr & 2 \\
Energy resolution (at 1 MeV) & $5 \%$ \\
Sensitivity for 1 ms burst detection, $\mathrm{cm}^{-2}$ & $\sim 3 \cdot 10^{-2}$ \\
Accuracy of burst source location & $\sim 10^{\circ}$ (for the brightest events) \\
Mass (for one detector module), $\mathrm{kg}$ & 5.5 \\
Information capacity, MByte/day & $\sim 500$ \\
Power consumption, W & 22.5
\end{tabular}

trigger. The calibration accuracy of each detector determines the systematic component of the TGF source error box.

The TGF source location accuracy for the GFM instrument was estimated by numerical simulations. The TGF source position in the instrument FOV was chosen, then gamma quantum position and interaction parameters in the detectors were simulated for the given distributions of energy and duration. Then, the number of detected gamma quanta in each detector was determined for a given energy range. Using the detectors outputs the TGF source coordinates were derived. Such simulations were repeated many times for bursts with given energy spectrum, flux values, and durations. The root mean square (RMS) of the detection angles of the restored source and hence, its error box, were also determined.

In order to estimate the direction reconstruction error, a $1 / 6$ th portion of the whole GFM field of view, i.e., crossed area of three detector FOVs, was bombarded by gammas, and 79 arrival directions were distributed evenly across this area. Three GFM detectors were treated separately for each arrival direction because the incident gamma flux was observed by them from different aspect angles. For each detector, all events with non-zero energy contribution to the $\mathrm{NaI}(\mathrm{Tl})$ and/or $\mathrm{CsI}(\mathrm{Tl})$ crystals were recorded. In order to make direction error estimates statistically robust, 20 independent samples were simulated for each arrival direction with different values of spectral index and fluence $(S)$.

The accuracy of TGF source localization depends on the brightness and hardness values of the TGF and the background level. The typical error box for very bright events with a fluence of $\sim 1 \mathrm{phot} / \mathrm{cm}^{2}$ is expected to be $\sim 5^{\circ}$, while for weak flares $\left(\sim 0.1 \mathrm{phot} / \mathrm{cm}^{2}\right)$, it may exceed $10^{\circ}$. For the expected satellite orbit altitude about $500-600 \mathrm{~km}$, it corresponds source area accuracy $\sim 40$ and $\sim 100 \mathrm{~km}$, respectively. It is quite rough positioning; however, it permits to connect the detected TGF event with a nearby thunderstorm area. More accurate TGF source location can be realized by using of position-sensitive tracking gamma ray spectrometer.

\section{Discussion and results}

Further progress in understanding of TLE and TGF phenomena can be achieved via the multi-wavelength and multi-messenger approach. The latter means the inclusion of hadronic signature of the phenomena in the process of understanding via the neutrons that are likely generated by high-altitude discharges, similar to the abovementioned neutron generation during thunderstorms (Enoto et al. (2017)).

Multi-wavelength observations of TLEs and TGFs will be realized with the use of above-presented combination of optical ultraviolet, $\mathrm{X}$-, and gamma-ray instruments. For clear thunderstorm identification, radio-frequency analyzer at least for wavelength band from several dozen kilohertz up to $15-20 \mathrm{MHz}$ should be added to the mission payload.

Taking into account the ranges of TGF, observed intensity and rate obtained by GRB Fermi (Tierney et al. (2013)), i.e., $0.05-0.5 \mathrm{count} / \mathrm{cm}^{2} / \mathrm{TGF}$ and $10^{-7}-$ $10^{-9} \mathrm{TGF} / \mathrm{h} / \mathrm{km}^{2}$, respectively, we can estimate the expected sensitivity of TGS instrument for minimal configuration $\left(256 \mathrm{~cm}^{2}\right)$ as $\sim 10$ counts per 1 TGF. Then, for polar orbit with $\sim 500 \mathrm{~km}$ altitude and about $\pm 30^{\circ}$ instrument field of view, the expected number of events will be about 1 event per day from near equatorial areas. Thus, for the time of experiment of about 3-5 years, the significant statistics of TGFs could be obtained. The sensitivity and, thus, the expected TGF statistics for GFM instrument is quite similar.

However, the crucial point in understanding of TGFs physical nature is confirming or refuting their direct connection with the given thunderstorm or lightning. For this goal, besides of the event fine timing, a good localization of the TGF source is also necessary. Of course, the angular resolution presented above for both GFM and TGS instruments, even for intensive TGFs, is not better than $\sim 10^{\circ}$ that scales to the space accuracy of TGF source localization of $\sim 100 \mathrm{~km}$ for the instruments flying at $500 \mathrm{~km}$ orbit. It is enough for TGF source identification with thunderstorm area, but it is insufficient to guarantee the identification with a given thunderstorm cloud. This latter task will demand an improvement of the angular resolution of suggested instruments by at least a factor of 10 . The solution for the localization problem can be achieved by applying the triangulation method for observations of the selected area with different satellites. As it was mentioned above, for this purpose, the Universat-SOCRAT multi-satellite mission could be added by some number of CubeSats, which should be launched with the main spacecraft into similar orbit for the joint observations of a given 
area. Sufficiently, light X-ray and gamma-ray detectors aimed for the TGF detection only, without spectrometer capabilities onboard the CubeSats will provide realization of the triangulation technique. By this, the necessity of maintaining of given distance between satellites is a separate technical problem. It can be solved using thrusters on micro-satellites.

The other result in TAE study could be connected to the possibilities of neutron detection. Simulation predicts detection of about two neutrons for event time $30 \mathrm{~ms}$ at the orbit with $450 \mathrm{~km}$ altitude, by the detector with $60 \mathrm{~cm}^{2}$ effective area (Drozdov et al. (2010); Grigoriev et al. (2010)). This result implies that detection of significant number of neutrons from TGFs is possible using the detector with effective area of about several hundred square centimeter. It means that above-described TGS instrument having minimal configuration of about $15 \mathrm{~cm}^{2}$ effective area should be scaled up just in a few times.

\section{Conclusions}

A new project Universat-SOCRAT is proposed by Lomonosov Moscow State University based on the previous experience of TLE and TGF measurements from space. The successful realization of the project will make it possible to create a prototype of the space system for monitoring and warning of the space hazards for both ongoing and planned space missions, including high-altitude stratospheric aircrafts for the first time in the space exploration history. For the latter, it is especially actual to control the electromagnetic transients in the upper Earth's atmosphere and space (GRBs, solar flares), because such transient phenomena in the atmosphere as TGFs may be dangerous for aircrafts and for the planned sub-orbital missions.

Multi-wavelength synchronous observations with moderate accuracy of localization of TGF and TLE events give the unique information about high-energy processes in the Earth's atmosphere. Principally, it allows identification of TGF source with concrete thunderstorm area and significantly improving of probability of TGF identification with lightning. The last is crucial for testing of TAE theoretical models. Simultaneous observation of TGFs and TLEs can give information about peculiarities of high-altitude discharge, which is the most probable cause of these phenomena. Measurements of UV and optical emission of TLEs with high temporal resolution will continue investigation of serial flashes observed in Vernov and Lomonosov experiments. It will give information about spectrum and space-time structure of the events in thunderstorm and far from the thunderstorm regions.

\section{Abbreviations}

ASIC: Application-specific integrated circuit; DUV: Detector of ultraviolet; GRB: Gamma-ray burst; IR: Infrared; HV: High voltage; MAPMT: Multi-anode photomultiplier tube MLT-S: Mini-lens telescope - spectrometer; MSU: Moscow State University; REB: Relativistic electron breakdown; RFB: Relativistic feedback discharge; RREA: Relativistic runaway electron avalanche; SiPM: Silicon photomultiplier; TAE: Transient atmospheric event; TGF: Terrestrial gamma-ray burst; TLE: Transient luminous event; UV: Ultraviolet; WWLLN: World Wide Lightning Location Network

\section{Acknowledgements}

Financial support for this work was provided by the Ministry of Education and Science of Russian Federation, Project \# RFMEFI60717X0175.

\section{Funding}

This work was supported by the Ministry of Education and Science of Russian Federation, Project \# RFMEFI60717X0175.

\section{Availability of data and materials}

Not applicable.

\section{Authors' contributions}

$\mathrm{MP}$ is a PI of Universat-SOCRAT experiment and author of general concept of the article. PK is a PI of the TUS Lomonosov and MLT-S Universat-SOCRAT experiments, and the main author of "Results of TLE observations in space experiments of Moscow State University" sections and "Instruments for TLE studies" of the article. SS is a PI of TGS and GFM Universat-SOCRAT experiments and the main author of "Introduction", "Results of TLE observations in space experiments of Moscow State University", "TGF observations in the

Universat-SOCRAT project", and "Discussion and results" sections of the article. $A B$ designed the MLT-S instrument electronic and is one of the authors of "Instruments for TLE studies" section intended for MLT-S description. VB designed the TGS instrument and is the author of "Tracking gamma-ray spectrometer" section of the article. AB is responsible for the data analysis and GFM instrument computer simulations, and is the author of "Gamma-ray flash monitor" section intended for GFM parameters. GG designed the UV spectrometer of MLT-S instrument electronic, and is one of the authors of "Instruments for TLE studies" section intended for MLT-S UV spectrometer description. Al is responsible for the analysis of neutron observations from the atmosphere, and is the author of the "The TLE and TGF study in other missions" section intended for neutron observations. MK carried out the TUS data analyses and collaborated with the corresponding author in the construction of the manuscript. IM designed the TDS instrument electronic and is one of the authors of "TGF observations in the Universat-SOCRAT project" section intended for TGS description. AM carried out the TGS computer simulations. AN provided the laboratory modeling of TGS units. PM collaborated in "The TLE and TGF study in other missions" section intended for optimization of TGF observations. VP is a project manager of Universat-SOCRAT experiment and is the author of "Concept of TLE and TGF observations in the Universat-SOCRAT project" section of the article. AP is an author of parts of "Introduction" and "The TLE and TGF study in other missions" sections intended for analysis of TGF study. YS carried out the laboratory modeling of MLT-S units. IY is an assistant of the project manager of Universat-SOCRAT experiment and is one of the authors of "Concept of TLE and TGF observations in the

Universat-SOCRAT project" section intended for the instrument description. All authors read and approved the final manuscript.

\section{Competing interests}

The authors declare that they have no competing interest.

\section{Publisher's Note}

Springer Nature remains neutral with regard to jurisdictional claims in published maps and institutional affiliations.

\section{Author details \\ ${ }^{1}$ Skobeltsyn Institute of Nuclear Physics, Lomonosov Moscow State University, 1(2), Leninskie gory, Moscow 119991, Russia. ${ }^{2}$ Physics Department, Lomonosov Moscow State University, 1(2), Leninskie gory, Moscow 119991 Russia. ${ }^{3}$ Space Research Institute, Russian Academy of Science, 84/32 Profsoyuznaya Str, Moscow 117997 Russia. ${ }^{4}$ National Research University Higher School of Economics, 20 Myasnitskaya Str, Moscow 101000 Russia.}

Received: 7 September 2018 Accepted: 25 March 2019

Published online: 16 April 2019 


\section{References}

Armstrong R, Suszcynsky D, Lyons W, Nelson T (2000) Multi-color photometric measurements of ionization and energies in sprites. Geophys Res Lett 27(5):653-656

Babich LP, Bochkov El, Donskoi EN, Kutsyk IM (2010) Source of prolonged bursts of high-energy gamma rays detected in thunderstorm atmosphere in japan at the coastal area of the sea of japan and on high mountaintop. J Geophys Res Space Phys 115(A9):A09317. https://doi.org/10.1029/ 2009JA015017

Babich LP, Bochkov El, Kutsyk IM, Roussel-Dupré RA (2010) Localization of the source of terrestrial neutron bursts detected in thunderstorm atmosphere. J Geophys Res Space Phys 115:00-28

Babich LP, Roussel-Dupré RA (2007) Origin of neutron flux increases observed in correlation with lightning. J Geophys Res Atmos 112(D13):D1330. https://doi.org/10.1029/2006JD008340

Blin-Bondil S, Dulucq F, Rabanal J, Dagoret-Campagne S, Tongbong J, Barrillon P, de La Taille C, Moretto C, Miyamoto H, Thienpont D (2014) Spaciroc3: A front-end readout asic for JEM-EUSO cosmic ray observatory. PoS 172(TIPP2014)

Bell TF, Pasko VP, Inan US (1995) Runaway electrons as a source of red sprites in the mesosphere. Geophys Res Lett 22(16):2127-2130

Budtz-Jorgensen C, Kuvvetli I, Skogseide Y, Ullaland K, Ostgaard N (2009) Characterization of CZT detectors for the asim mission. IEEE Trans Nucl Sci 56(4):1842-1847

Blanc E, Lefeuvre F, Roussel-Dupré R, Sauvaud J (2007) TARANIS: A microsatellite project dedicated to the study of impulsive transfers of energy between the earth atmosphere, the ionosphere, and the magnetosphere. Adv Space Res 40(8):1268-1275

Boccippio D, R. Williams E, Heckman S, Lyons W, T. Baker I, Boldi R (1995) Sprites, elf transients, and positive ground strokes. Science 269:1088-1091

Boeck WL, Vaughan OH, Blakeslee RJ, Vonnegut B, Brook M, McKune J (1995) Observations of lightning in the stratosphere. J Geophys Res Atmos 100(D1):1465-1475

Bogomolov V, Panasyuk M, Svertilov S, Bogomolov A, Garipov G, lyudin A, Klimov P, Klimov S, Mishieva T, Minaev PY, et al. (2017) Observation of terrestrial gamma-ray flashes in the RELEC space experiment on the Vernov satellite. Cosm Res 55(3):159-168

Briggs MS, Connaughton V, Wilson-Hodge C, Preece RD, Fishman GJ, Kippen RM, Bhat P, Paciesas WS, Chaplin VL, Meegan CA, et al. (2011) Electronpositron beams from terrestrial lightning observed with Fermi GBM. Geophys Res Lett 38(2):L02808. https://doi.org/10.1029/2010GL046259

Briggs MS, Xiong S, Connaughton V, Tierney D, Fitzpatrick G, Foley S, Grove JE, Chekhtman A, Gibby M, Fishman GJ, et al. (2013) Terrestrial gamma-ray flashes in the Fermi era: Improved observations and analysis methods. J Geophys Res Space Phys 118(6):3805-3830

Briggs MS, Wersinger JM, Fogle Jr. M, Biaz S, Jenke P (2015) TRYAD: a pair of CubeSats to measure terrestrial gamma-ray flash beams. AGU Fall Meet Abstr:33-0481

Capel F, Belov A, Casolino M, Klimov P (2018) Mini-EUSO: A high resolution detector for the study of terrestrial and cosmic UV emission from the International Space Station. Adv Space Res 62(10):2954-2965. https://doi. org/10.1016/j.asr.2017.08.030. Origins of Cosmic Rays

Carlson B, Lehtinen NG, Inan US (2010) Neutron production in terrestrial gamma ray flashes. J Geophys Res Space Phys 115(A4):A00E19. https://doi. org/10.1029/2009JA014696

Chern J, Hsu R, Su H-T, Mende S, Fukunishi H, Takahashi Y, Lee L-C (2003) Global survey of upper atmospheric transient luminous events on the ROCSAT-2 satellite. J Atmos Solar-Terrestrial Phys 65(5):647-659

Christian H, Frost R, Gillaspy P, Goodman S, Vaughan Jr O, Brook M, Vonnegut B, Orville R (1983) Observations of optical lightning emissions from above thunderstorms using U-2 aircraft. Bull Am Meteorol Soc 64(2):120-123

Christian H, Blakeslee R, Goodman S, Mach D, Stewart M, Buechler D, Koshak W, Hall J, Boeck W, Driscoll K, et al. (1999) The lightning imaging sensor. In: Proc. 11 th Int. Conf. on Atmospheric Electricity, Guntersville, AL, ICAE. NASA. pp 746-749

Cummer SA, Briggs MS, Dwyer JR, Xiong S, Connaughton V, Fishman GJ, Lu G, Lyu F, Solanki R (2014) The source altitude, electric current, and intrinsic brightness of terrestrial gamma ray flashes. Geophys Res Lett 41(23):8586-8593

Drozdov A, Amelushkin A, Bratolyubova-Tsulukidze L, Churilo I, Grigoriev A Grigoryan O, ludin D, Mareev E, Nechaev O, Petrov V (2010) Experiment based on spacesuit "Orlan-M": Neutron fluxes from thunderstorms. J Geophys Res Space Phys 115(A8):A00E51
Dwyer JR (2007) Relativistic breakdown in planetary atmospheres. Phys Plasmas 14(4):042901

Dwyer JR, Grefenstette BW, Smith DM (2008) High-energy electron beams launched into space by thunderstorms. Geophys Res Lett 35(2):L02815. https://doi.org/10.1029/2007GL032430

Dwyer JR, Smith DM, Cummer SA (2012) High-energy atmospheric physics: Terrestrial gamma-ray flashes and related phenomena. Space Sci Rev 173(1-4):133-196

Enoto T, Wada Y, Furuta Y, Nakazawa K, Yuasa T, Okuda K, Makishima K, Sato M, Sato Y, Nakano T, et al. (2017) Photonuclear reactions triggered by lightning discharge. Nature 551(7681):481

Farges T, Hébert P, Le Mer-Dachard F, Ravel K, Gaillac S (2017) Microcameras and photometers (MCP) on board the TARANIS satellite. In: AGU Fall Meeting Abstracts. abstract \#AE21A-04

Fisher JR (1990) Upward discharges above thunderstorms. Weather 45(12):451-452

Fishman GJ, Bhat P, Mallozzi R, Horack J, Koshut T, Kouveliotou C, Pendleton G, Meegan C, Wilson R, Paciesas W, et al. (1994) Discovery of intense gamma-ray flashes of atmospheric origin. Science 264(5163):1313-1316

Fukunishi H, Takahashi Y, Kubota M, Sakanoi K, Inan US, Lyons WA (1996) Elves: Lightning-induced transient luminous events in the lower ionosphere. Geophys Res Lett 23(16):2157-2160. https://doi.org/10.1029/96GL01979

Fuquay DM, Baughman RG, Taylor AR, Hawe RG (1967) Characteristics of seven lightning discharges that caused forest fires. J Geophys Res 72(24):6371-6373

Garipov G, Khrenov B, Panasyuk M, Tulupov V, Shirokov A, Yashin I, Salazar H (2005) UV radiation from the atmosphere: results of the msu "Tatiana" satellite measurements. Astropart Phys 24(4-5):400-408

Garipov G, Panasyuk M, Rubinshtein I, Tulupov V, Khrenov B, Shirokov A, Yashin I, Salazar H (2006) Ultraviolet radiation detector of the MSU research educational microsatellite Universitetskii-Tat'yana. Instrum Exp Tech 49(1):126-131

Garipov G, Khrenov B, Klimov P, Klimenko V, Mareev E, Martines O, Mendoza E, Morozenko V, Panasyuk M, Park I, et al. (2013) Global transients in ultraviolet and red-infrared ranges from data of Universitetsky-Tatiana-2 satellite. J Geophys Res Atmos 118(2):370-379

Grigoriev A, Grigoryan O, Drozdov AY, Malyshkin Y, Popov Y, Mareev E, ludin D (2010) Thunderstorm neutrons in near space: analyses and numerical simulation. J Geophys Res Space Phys 115(A8):A00E52. https://doi.org/10. 1029/2009JA014870

Gurevich A, Milikh G, Roussel-Dupre R (1992) Runaway electron mechanism of air breakdown and preconditioning during a thunderstorm. Phys Lett $A$ 165(5-6):463-468

Iwanowska J, Swiderski L, Szczesniak T, Sibczynski P, Moszynski M, Grodzicka M, Kamada K, Tsutsumi K, Usuki Y, Yanagida T, et al. (2013) Performance of cerium-doped Gd3Al2Ga3012 (GAGG: Ce) scintillator in gamma-ray spectrometry. Nucl Inst Methods Phys Res Sect A: Accelerators, Spectrometers, Detectors Assoc Equip 712:34-40

Iyudin A, Bogomolov V, Galkin V, Golovanov I, Krasnov A, Markelova A, Markelov I, Morgunova Y, Osedlo V, Panasyuk M, et al. (2015) Instruments to study fast neutrons fluxes in the upper atmosphere with the use of high-altitude balloons. Adv Space Res 56(10):2073-2079

Khrenov B, Klimov P, Panasyuk M, Sharakin S, Tkachev L, Zotov MY, Biktemerova S, Botvinko A, Chirskaya N, Eremeev V, et al. (2017) First results from the TUS orbital detector in the extensive air shower mode. J Cosmol Astropart Phys 2017(09):006

Klimov P, Garipov G, Khrenov B, Morozenko V, Barinova V, Bogomolov V, Kaznacheeva M, Panasyuk M, Saleev K, Svertilov S (2017) Vernov satellite data of transient atmospheric events. J Appl Meteorol Climatol 56(8):2189-2201

Klimov P, Panasyuk M, Khrenov B, Garipov G, Kalmykov N, Petrov V, Sharakin S, Shirokov A, Yashin I, Zotov M. Y, et al (2017) The TUS detector of extreme energy cosmic rays on board the Lomonosov satellite. Space Sci Rev 212(3-4):1687-1703

Klimov P, Kaznacheeva M, Khrenov B, Garipov G, Bogomolov V, Panasyuk M, Svertilov S, Cremonini R (2018) UV transient atmospheric events observed far from thunderstorms by the Vernov satellite. IEEE Geosci Remote Sens Lett 15(8):1139-1143

Lehtinen NG, Bell TF, Pasko V, Inan U (1997) A two-dimensional model of runaway electron beams driven by quasi-electrostatic thundercloud fields. Geophys Res Lett 24(21):2639-2642 
Lyons WA (1994) Characteristics of luminous structures in the stratosphere above thunderstorms as imaged by low-light video. Geophys Res Lett 21(10):875-878

Mazets EP, Golenetskii SV (1981) Recent results from the gamma-ray burst studies in the KONUS experiment. Astrophys Space Sci 75:47-81

Milikh G, Valdivia J, Papadopoulos K (1998) Spectrum of red sprites. J Atmos Solar-Terrestrial Phys 60(7-9):907-915

Minaev PY, Pozanenko A, Molkov S, Grebenev S (2014) Catalog of short gamma-ray transients detected in the SPI/INTEGRAL experiment. Astron Lett 40(5):235-267

Minaev P, Pozanenko A (2018) Search for short transient gamma-ray events in SPI experiment onboard INTEGRAL: The algorithm and results. In: Kalinichenko L, Manolopoulos Y, Malkov O, Skvortsov N, Stupnikov S, Sukhomlin V (eds). Data Analytics and Management in Data Intensive Domains. Springer, Cham. pp 128-138

Moos RH, Moos BS (2006) Rates and predictors of relapse after natural and treated remission from alcohol use disorders. Addiction 101(2):212-222

Neubert T, Kuvvetli I, Budtz-Jørgensen C, Østgaard N, Reglero V, Arnold N (2006) The atmosphere-space interactions monitor (ASIM) for the international space station. In: ILWS (International Living With a Star) Workshop. pp 19-20

Orville RE, Henderson RW (1984) Absolute Spectral Irradiance Measurements of Lightning from 375 to $880 \mathrm{~nm}$. J Atmos Sci 41:3180-3180

Østgaard N, Gjesteland T, Stadsnes J, Connell P, Carlson B (2008) Production altitude and time delays of the terrestrial gamma flashes: Revisiting the burst and transient source experiment spectra. J Geophys Res Space Phys 113(A2):A02307. https://doi.org/10.1029/2007JA012618

Panasyuk M, Svertilov S, Bogomolov V, Garipov G, Barinova V, Bogomolov A, Veden'kin N, Golovanov I, lyudin A, Kalegaev V, et al (2016a) Experiment on the Vernov satellite: Transient energetic processes in the Earth's atmosphere and magnetosphere. Part I: Description of the experiment. Cosm Res 54(4):261-269

Panasyuk M, Svertilov S, Bogomolov V, Garipov G, Barinova V, Bogomolov A, Veden'kin N, Golovanov I, lyudin A, Kalegaev V, et al. (2016b) Experiment on the Vernov satellite: transient energetic processes in the Earth's atmosphere and magnetosphere. Part II, First results. Cosm Res 54(5):343-350

Panasyuk M, Podzolko M, Kovtyukh A, Brilkov I, Vlasova N, Kalegaev V, Osedlo V Tulupov V, Yashin I (2015) Operational radiation monitoring in near-earth space based on the system of multiple small satellites. Cosm Res 53(6):423-429

Panasyuk M, Podzolko M, Kovtyukh A, Brilkov I, Vlasova N, Kalegaev V, Osedlo V Tulupov V, Yashin I (2017) Optimization of measurements of the Earth's radiation belt particle fluxes. Cosm Res 55(2):79-87

Pasko VP, Yair Y, Kuo C-L (2012) Lightning related transient luminous events at high altitude in the Earth's atmosphere: phenomenology, mechanisms and effects. Space Sci Rev 168:475-516. https://doi.org/10.1007/s11214011-9813-9

Roberts OJ, Fitzpatrick G, Stanbro M, McBreen S, Briggs MS, Holzworth RH, Grove JE, Chekhtman A, Cramer ES, Mailyan BG (2018) The first Fermi-GBM terrestrial gamma ray flash catalog. J Geophys Res Space Phys 123(5):4381-4401. https://doi.org/10.1029/2017JA024837. https:// agupubs.onlinelibrary.wiley.com/doi/pdf/10.1029/2017JA024837

Sadovnichy V, Panasyuk M, Bobrovnikov SY, Vedenkin N, Vlasova N, Garipov G, Grigorian O, Ivanova T, Kalegaev V, Klimov P, et al. (2007) First results of investigating the space environment onboard the Universitetskii-Tatyana satellite. Cosm Res 45(4):273-286

Sadovnichy V, Panasyuk M, Yashin I, Barinova V, Veden'kin N, Vlasova N, Garipov G, Grigoryan O, Ivanova T, Kalegaev V, et al (2011) Investigations of the space environment aboard the Universitetsky-Tat'yana and Universitetsky-Tat'yana-2 microsatellites. Sol Syst Res 45(1):3-29

Sadovnichii V, Panasyuk M, Amelyushkin A, Bogomolov V, Benghin V, Garipov G, Kalegaev V, Klimov P, Khrenov B, Petrov V, et al (2017) "Lomonosov" satellite-space observatory to study extreme phenomena in space. Space Sci Rev 212(3-4):1705-1738

Sarria D, Lebrun F, Blelly P-L, Chipaux R, Laurent P, Sauvaud J-A, Prech L, Devoto P, Pailot D, Baronick J-P, et al (2017) TARANIS XGRE and IDEE detection capability of terrestrial gamma-ray flashes and associated electron beams. Geosci Instrum Methods Data Syst 6(2):239-256

Sato M, Adachi T, Ushio T, Morimoto T, Kikuchi M, Kikuchi H, Suzuki M, Yamazaki A, Takahashi Y, Ishida R, Sakamoto Y, Yoshida K, Hobara Y (2017) Sprites identification and their spatial distributions in JEM-GLIMS nadir observations. Terr Atmos Ocean Sci 28:545-561. https://doi.org/10.1029/ 96GL01979

Sharkov EA, Kuzmin AV, et al. (2018) Space experiment "Convergence": scientific objectives, on-board equipment, methods of reverse problems. Izv. Atm. Ocean Phys 54(9):71-96

Shibata S (1994) Propagation of solar neutrons through the atmosphere of the Earth. J Geophys Res Space Phys 99(A4):6651-6665

Smith DM, Lopez LI, Lin RP, Barrington-Leigh CP (2005) Terrestrial gamma-ray flashes observed up to $20 \mathrm{MeV}$. Science 307(5712):1085-1088

Svertilov S, Panasyuk M, Bogomolov V, Amelushkin A, Barinova V, Galkin V, lyudin A, Kuznetsova E, Prokhorov A, Petrov V, et al (2018) Wide-field gamma-spectrometer BDRG: GRB monitor on-board the Lomonosov mission. Space Sci Rev 214(1):8

Tavani M, Marisaldi M, Labanti C, Fuschino F, Argan A, Trois A, Giommi P, Colafrancesco S, Pittori C, Palma F, et al (2011) Terrestrial gamma-ray flashes as powerful particle accelerators. Phys Rev Lett 106(1):018501

Tierney D, Briggs M, Fitzpatrick G, Chaplin V, Foley S, McBreen S, Connaughton V, Xiong S, Byrne D, Carr M, et al (2013) Fluence distribution of terrestrial gamma ray flashes observed by the Fermi Gamma-ray Burst Monitor. J Geophys Res Space Phys 118(10):6644-6650

Ushio T, Sato M, Morimoto T, Suzuki M, Kikuchi H, Yamazaki A, Takahashi Y, Hobara Y, Inan U, Linscott I, et al (2011) The global lightning and sprite measurement (GLIMS) mission on International Space Station. IEEJ Trans Fundam Mater 131(12):971-976

Vaughan O, Vonnegut B (1989) Recent observations of lightning discharges from the top of a thundercloud into the clear air above. J Geophys Res Atmos 94(D11):13179-13182

Vedenkin N, Garipov G, Klimov P, Klimenko V, Mareev E, Martinez O, Morozenko V, Park I, Panasyuk M, Ponce E, et al (2011) Atmospheric ultraviolet and red-infrared flashes from Universitetsky-Tatiana-2 satellite data. J Exp Theor Phys 113(5):781-790

Winckler J, Lyons W, Nelson T, Nemzek R (1996) New high-resolution ground-based studies of sprites. J Geophys Res Atmos 101(D3):6997-7004

Zoglauer AC (2005) First light for the next generation of compton and pair telescopes. PhD thesis. Technische Universität München

Zoglauer A, Andritschke R, Schopper F (2006) MEGAlib-the medium energy gamma-ray astronomy library. New Astron Rev 50(7-8):629-632

\section{Submit your manuscript to a SpringerOpen ${ }^{\circ}$ journal and benefit from:}

- Convenient online submission

- Rigorous peer review

- Open access: articles freely available online

- High visibility within the field

- Retaining the copyright to your article

Submit your next manuscript at springeropen.com 